\title{
“DRILL, BABY, DRILL": LA POSICIÓN DE ESTADOS UNIDOS ANTE EL RETO AMBIENTAL DE LA EXPLORACIÓN Y EXPLOTACIÓN DE HIDROCARBUROS EN EL ÁRTICO*
}

\author{
MAR CAMPINS ERITJA \\ Profesora Titular de Universidad (acreditada al cuerpo de Catedráticos por ANECA) \\ Departamento Derecho Penal y Criminología y Derecho Internacional Público y \\ Relaciones Internacionales \\ Titular de la Cátedra Jean Monnet de Derecho Ambiental de la Unión Europea \\ Universitat de Barcelona \\ mcampins@ub.edu
}

Recibido: 14 de septiembre de 2017/ Aceptado: 14 de noviembre de 2017

RESUMEN: El presente trabajo examina la posición de Estados Unidos con respecto de la exploración y explotación de las reservas de petróleo y gas estimadas en las zonas árticas bajo su jurisdicción. Después de una breve introducción a la región ártica, el trabajo destaca el alcance de esta actividad y su impacto sobre el medio ambiente ártico. Seguidamente y como marco general, el trabajo describe los principales rasgos de la estrategia de Estados Unidos en la región ártica, y a partir de aquí, se centra en el examen de dos aspectos que reflejan una política energética estadounidense fluctuante frente a las exigencias de protección de un medio ambiente tan vulnerable como el del Ártico. De una parte, se presenta el debate sobre las competencias de las autoridades federales y estatales para administrar y supervisar los programas de arrendamiento para la exploración y explotación mar adentro de hidrocarburos en el Ártico. De la otra, se

\footnotetext{
* Este era el lema que utilizó la gobernadora de Alaska y candidata republicana a la vicepresidencia, Sarah Palin, durante el debate vicepresidencial en octubre de 2008 para expresar su apoyo a las actividades de perforación de hidrocarburos en el estado de Alaska. Este trabajo se ha elaborado en el marco del proyecto de investigación La incidencia del deshielo de la región ártica en la recomposición del espacio geo-energético mundial, financiado por el Institut Català Internacional de la Pau (ICIP), 2015RICIP00005. Asimismo, se ha beneficiado de una estancia de investigación Fulbright Visiting Scholar en el marco del Programa de estancias de profesores e investigadores senior, incluido el programa "Salvador de Madariaga" (PR2015-00176) en el Washington College of Law de American University.
} 
examina la política de limitación progresiva de estos programas en el periodo de presidencia de Barack Obama, ahora en proceso de revisión por la nueva administración americana. Ambas cuestiones resultan especialmente relevantes en el momento actual, significado por la apertura de del gobierno federal a la exploración y explotación de estos recursos como base de su política energética.

RESUM: Aquest treball examina la posició dels Estats Units respecte a l'exploració i explotació de les reserves estimades de petroli i gas en les zones àrtiques sota la seva jurisdicció. Després d'una breu introducció a la regió àrtica, el treball destaca l'abast d'aquesta activitat i el seu impacte sobre el medi ambient àrtic. Seguidament i com a marc general, el treball presenta els trets principals de l'estratègia d'Estats Units a la regió àrtica. A partir d'aquí, el treball es centra en l'examen de dos aspectes que reflecteixen una política energètica nord-americana força fluctuant davant les exigències de protecció d'un entorn tan vulnerable com el de l'Àrtic. D'una banda, el debat sobre les competències de les autoritats federals i estatals per administrar i supervisar els programes d'arrendament per a l'exploració i explotació mar endins d'hidrocarburs a l'Àrtic. De l'altra, la política de limitació progressiva d'aquests programes d'arrendament iniciada per l'administració del President Obama, ara en procés de revisió per la nova administració americana del President Trump. Les dues qüestions resulten especialment rellevants en el moment actual, caracteritzat per l'obertura del govern federal dels Estats Units a l'exploració i explotació d'aquests recursos com a base de la seva política energètica.

ABSTRACT: This paper analyses the position of the United States with respect to the exploration and exploitation of the Arctic oil and gas reserves under its jurisdiction. After a brief introduction to the Arctic region, the article highlights the scope and impact of this activity on the Arctic environment, as well as the main features of US strategy in the Arctic region. The article goes on to focus on two issues reflecting a fluctuating US energy policy in the face of environmental protection requirements in such a vulnerable region. On the one hand, it presents the debate on federal and state authorities' legal competences to handle leasing programs for offshore exploration and exploitation of Arctic hydrocarbons. On the other hand, it explores the US policy of progressive limitation of these programs under President Obama's administration, now in the process of being reversed by President Trump's administration. Both issues are 
currently of great relevance since the opening of these resources to exploration and exploitation is to be considered as a core feature of US energy policy under President Trump's administration.

PALABRAS CLAVE: Océano Ártico - Estados Unidos - Hidrocarburos Protección del medio ambiente.

PARAULES CLAU: Oceà Àrtic — Estats Units — Hidrocarburs — Protecció del medi ambient.

KEYWORDS: Arctic Ocean - United States - Hydrocarbons - Environmental protection.

SUMARIO: I. Introducción. II. Los retos ambientales que afronta la región ártica en relación con la exploración y explotación de hidrocarburos.1. La quimera del oro negro. La explotación de hidrocarburos en el Ártico. 2. La realidad del riesgo ambiental. Implicaciones de una contaminación por hidrocarburos en el Ártico. III. Los intereses estratégicos de Estados Unidos en el Ártico y su política en relación a la exploración y explotación de sus hidrocarburos. 1. La posición de Estados Unidos en el Ártico. 2. El conflicto entre el gobierno federal y el estado de Alaska en torno a la exploración y explotación mar adentro de petróleo ártico. 3. El lento e inacabado proceso de limitación de los arrendamientos para la exploración y explotación mar adentro en el Ártico. IV. Consideraciones finales. V. Bibliografía.

\section{INTRODUCCIÓN}

La región ártica, un área de cerca de 14,5 millones de $\mathrm{km}^{2}$ en el que se asientan no más de cuatro millones de habitantes se enfrenta actualmente a una serie de retos de gran complejidad que determinarán su futuro desarrollo en múltiples dimensiones ${ }^{1}$. Además de sus especiales condiciones climatológicas, la región ártica se caracteriza por la presencia de la banquisa polar, lo que no impide la presencia de una variada y rica fauna, además de contar con importantes recursos minerales e hidrocarburos. El problema más acuciante en la región lo constituye actualmente el fenómeno del cambio climático. El último informe del Panel Intergubernamental sobre el Cambio Climático (PICC) confirma la pérdida de masa del manto de hielo a un ritmo más rápido entre 2002 y 2011 y un aumento de las temperaturas del permafrost desde principios de la década de 1980, lo que ha contribuido en más del $40 \%$ en el aumento del nivel del mar

\footnotetext{
${ }^{1}$ OSHERENKO, G.-YOUNG, O., The Age of the Arctic: Hot Conflicts and Cold realities, Cambridge University Press, Cambridge, 2005, pp.168 y ss.
} 
entre 2003 y $2008^{2}$. El PICC considera "muy probable que la superficie media anual del hielo marino del Ártico haya disminuido durante el período 1979-2012 en un rango del $3,5 \%$ al $4,1 \%$ por decenio" debido a la influencia antropogena. Sus proyecciones señalan la gravedad de esta situación en un entorno con pocas opciones de adaptación e indican que la región del Ártico seguirá calentándose más rápidamente que la media global, con una disminución del hielo marino en todos los escenarios. Apuntan también la probabilidad de que, antes de mediados de siglo, el océano Ártico esté casi libre de hielo en el mes de septiembre, con una disminución del permafrost entre el 37\% y el $81 \%{ }^{3}$.Esta situación constituye lo que ya se conoce como el new normal de esta región, una expresión que se utiliza para reflejar el cambio a una nueva normalidad de hielo marino ${ }^{4}$. Incide en las condiciones de la atmósfera, el océano y la tierra, manifestándose en cambios físicos y biológicos importantes y persistentes que afectan a todo el planeta.

Las consecuencias más evidentes de esta situación se identifican con una mayor viabilidad de las vías de navegación árticas entre Europa y Asia, el acceso a nuevas pesquerías y la posibilidad de la exploración y explotación del petróleo, el gas y las reservas minerales ${ }^{5}$, todos ellos factores que aumentan la presión y el potencial de contaminación en esta zona. La alteración de las condiciones ambientales debido al fenómeno del cambio climático no sólo conlleva importantes pérdidas para el conjunto del medio ambiente, sino que a su vez facilita o intensifica algunas de las actividades más contaminantes en el Ártico, como el desarrollo de la industria petrolera y gasística. Ante la necesidad de garantizar la seguridad energética y atender la creciente demanda mundial de energía, junto con la relativa estabilidad política de la región y los avances tecnológicos que posibilitan la explotación de estos recursos, la estimación de

\footnotetext{
${ }^{2}$ Arctic Monitoring and Assessment Programme, 2011 Assessment of the Impacts of Climate Change on Snow, Water, Ice and Permafrost in the Arctic (SWIPA), 2011, disponible online en http://www.amap.no/swipa

${ }^{3}$ PACHAURI, R.K., MEYER, L.A., Cambio climático 2014: Informe de síntesis. Contribución de los Grupos de trabajo I, II y III al Quinto Informe de Evaluación del Grupo Intergubernamental de Expertos sobre el Cambio Climático, IPCC, Ginebra, 2014, especialmente p. 44, 51, 64, 66, 75 y 77, disponible online en https://www.ipcc.ch/pdf/assessment-report/ar5/syr/SYR_AR5_FINAL_full_es.pdf.

${ }^{4}$ JEFFRIES, M.O., OVERLAND, J.E, PEROVICH,D.K., "The Arctic shifts to a new normal", Physics Today, Vol. 66(10), 2013, pp. 35-40, p. 36 y 39, disponible online en http://dx.doi.org/10.1063/PT.3.2147.

${ }^{5}$ IHS Incorporated, International Petroleum Exploration and Production Database (IHS Incorporated, Englewood, CO, 2007) citado en GAUTIER, D. et al. "Assessment of Undiscovered Oil and Gas in the Arctic", Science, Vol. 324, 2009, pp. 1175-1179, disponible online en http://www.sciencemag.org/cgi/content/full/sci;324/5931/1175? maxtoshow=\&hits=10\&RESULTFORM $\mathrm{AT}=\&$ fulltext=Arctic+AND+oil+AND+gas\&searchid=1\&FIRSTINDEX=0\&resourcetype=HWCIT.
} 
importantes reservas de hidrocarburos ha estado en la vanguardia del reciente interés en la región.

En este contexto, los cinco Estados ribereños del Océano Ártico, Estados Unidos, Canadá, la Federación Rusa, Noruega y Groenlandia (Dinamarca) tienen en sus manos la clave para el desarrollo futuro en el Ártico. Estos son también los cinco Estados parte en el primer acuerdo con dimensión ártica, el Acuerdo sobre la conservación de los osos polares de $1973^{6}$, y los cinco Estados que han firmado la Declaración de Ilulissat resultado de la Conferencia celebrada en Ilulissat (Groenlandia) entre el 27 y el 29 de mayo de $2008^{7}$. Interesados en el diseño de un sistema de gestión ajustado a sus intereses, estos Estados priorizan la dimensión nacional de los recursos árticos y aunque todos ellos han mostrado una cierta apertura en lo que respecta a la cooperación internacional, tienen una clara preferencia por los foros de negociación restringidos que respaldan sus propósitos ${ }^{8}$.

El presente trabajo examina la posición de Estados Unidos con respecto de la exploración y explotación de las reservas de petróleo y gas estimadas en los espacios

6 Texto en IUCN/SSC Polar Bear Specialist Group, disponible online en http://pbsg.npolar.no/en/agreements/agreement1973.html.

7 The Ilulissat Declaration, Arctic Ocean Conference, Ilulissat, Greenland, 27-29 May 2008, disponible online en http://www.oceanlaw.org/downloads/arctic/Ilulissat_Declaration.pdf. A este respecto es relevante mencionar que la Declaración de Ilulissat refleja claramente el celo de los cinco Estados árticos en la preservación de sus derechos soberanos sobre esta región. El sistema articulado mediante este instrumento crea un foro de negociación informal que funciona de hecho de forma paralela al Consejo Ártico y que en cierto modo puede interferir en el desarrollo de sus objetivos. A diferencia de éste, prioriza la cooperación exclusivamente entre los cinco Estados ribereños del Océano Ártico a los que limita la participación, abriéndose solo parcialmente a los otros tres Estados árticos (Finlandia, Islandia y Suecia) y obviando la presencia de los representantes de los pueblos indígenas del Ártico.

${ }^{8}$ ROSSI, Ch.R., "The club within the club: The challenge of a soft law framework in a global Arctic context", The Polar Journal, Vol. 5 (1), pp. 8-34, pp. 11 y 25 y ss. Vid. También, MOLENAAR, E.J., “Arctic Marine Shipping: Overview of the International Legal Framework. Gaps and Option”, Journal of Transnational Law \& Policy, Vol. 18, 2009, pp.289-325, pp. 298 y ss., 323 y ss.; YOUNG, O., "Arctic in Play: Governance in a Time of Rapid Change", The International Journal of Marine and Coastal Law, Vol. 24, 2009, pp. 423-442, p. 428 y ss.; RAYFUSE, R., "Melting Moments: The Future of Polar Oceans Governance in a Warming World", RECIEL, vol. 16(2), 2007, pp. 196-216; CASPER, K.N., "Oil and Gas Development in the Arctic: Softening of Ice Demands Hardening of International Law", Natural Resources Journal, Vol. 49, 2009, pp. 825-881. Entre la doctrina española, Vid., PAREJA, P., "El Ártico como espacio de cooperación: Evolución, contenido y limites", en SOBRINO HEREDIA, J.M., (Ed.), Mares y océanos en un mundo de cambio: Tendencias jurídicas, actores y factores, 2007, pp. 883-906; ALCAIDE FERNÁNDEZ, J., CINELLI, C., "La cuestión ártica y el Derecho Internacional", Revista Española de Derecho Internacional, Vol. 61 (2), 2009, pp. 381-409; CONDE PÉREZ, E., "Geopolítica del Ártico: El derecho internacional ante los retos del cambio climático en la región”, Cursos de Derecho Internacional y Relaciones Internacionales de Vitoria Gasteiz, 2014, pp. 99-160; CINELLI, C., "La protección del medio ambiente ártico: Desarrollos recientes”, en Sobrido Prieto, M. (Ed.), Espacios polares y cambio climático: Desafíos jurídico-internacionales, Tirant lo Blanch, 2017, pp. 185-210; SOBRIDO PRIETO, M., "La gobernanza del Ártico", en Sobrido Prieto, M. (Ed.), Espacios polares y cambio climático: Desafios jurídico-internacionales, Tirant lo Blanch, 2017, pp. 105-138. 
árticos bajo su jurisdicción. Entre los Estados ribereños, Estados Unidos -miembro fundacional del Consejo Ártico además de signatario de la Declaración de Ilulissat de 2008- es el que ha tenido una política ártica más dispar, evolucionando desde el más absoluto desinterés inicial hasta proclamarse adalid en las acciones de protección del entorno ártico, para acabar posicionándose en este último año como uno de los promotores de la explotación indiscriminada de los recursos de hidrocarburos.

En primer lugar, el trabajo refiere el alcance de esta industria en Estados Unidos y destaca su impacto sobre el medio ambiente ártico, un aspecto que ha dominado ampliamente los debates relativos al desarrollo de la región ártica. Como marco general, el trabajo describe seguidamente los principales rasgos de la estrategia de Estados Unidos en la región ártica, y a partir de aquí, se centra en el examen de dos aspectos que reflejan una política energética estadounidense fluctuante frente a las exigencias de protección de un medio ambiente tan vulnerable como el del Ártico. De una parte, se presenta el debate sobre las competencias de las autoridades federales y la disminución del papel de las autoridades estatales afectadas para administrar y supervisar los programas de arrendamiento para la exploración y explotación mar adentro (offshore) de hidrocarburos en el Ártico. De la otra, se centra en la política de limitación progresiva de estos programas en el periodo de presidencia de Barack Obama, ahora en proceso de revisión por parte de su sucesor Donald Trump. Ambas cuestiones resultan especialmente relevantes en el momento actual, significado por la nueva apertura del gobierno federal a la exploración y explotación de estos recursos como base de su política energética.

\section{LOS RETOS AMBIENTALES QUE AFRONTA LA REGIÓN ÁRTICA EN RELACIÓN CON LA EXPLORACIÓN Y EXPLOTACIÓN DE HIDROCARBUROS}

En 2008 el US Geological Service (USGS) valoró unas reservas de petróleo y gas en la plataforma continental al norte del Circulo Ártico que podrían llegar hasta los 90.000 millones de barriles de petróleo y 44.000 millones de barriles de gas natural líquido, además de 47,2 billones de $\mathrm{m}^{3}$ de gas natural, lo que constituye el $13 \%$ del mercado mundial de petróleo, además del 30\% del gas natural y el $20 \%$ de gas licuado por 
descubrir ${ }^{9}$. Estos recursos se encuentran principalmente en la plataforma continental ártica de los cinco Estados ribereños, lo que ha despertado el interés por esta región ${ }^{10}$. Sin embargo, la explotación mar adentro de estos recursos en el Océano Ártico tiene, sin duda, una incidencia muy significativa en la calidad del medio ambiente ártico y afecta directamente a la fauna y la flora ártica y a los ecosistemas a los que pertenecen, así como a las comunidades locales que dependen de los mismos.

\section{La quimera del oro negro: La explotación de hidrocarburos en el Ártico}

Aunque la prospección, perforación y explotación de hidrocarburos en la región ártica han suscitado un mayor interés entre la opinión pública sólo recientemente, estas actividades se han venido desarrollando desde finales de los años sesenta. En 1968, ARCO y Standard Oil perforaron un pozo en una de las bolsas de petróleo más grandes

\footnotetext{
${ }^{9}$ BIRD, K.J. et al., Circum-Arctic Resource Appraisal: Estimates of Undiscovered Oil and Gas North of the Arctic Circle, U.S. Geological Survey, 2008, disponible online en http://pubs.usgs.gov/fs/2008/3049.

${ }^{10}$ Noruega, la Federación Rusa, Estados Unidos, Canadá y Groenlandia tienen bajo su jurisdicción el $80 \%$ del territorio y de los espacios marinos donde se encuentran estos recursos. Aunque este artículo no analiza las cuestiones relativas a la delimitación de los espacios marinos, cabe señalar que todos los Estados árticos, excepto Estados Unidos, que no es parte en el Convenio del Derecho del Mar, han solicitado a la Comisión de Limites de la Plataforma Continental la ampliación de los límites de su plataforma continental. La Federación Rusa fue el primer Estado de la región ártica que presentó, el 20 de diciembre de 2001 respecto a las cordilleras submarinas Alfa-Mendelev y Lomonosov. La Comisión respondió a dicha petición solicitando información adicional. La respuesta rusa fue plantear dos peticiones distintas - una reclamación sobre el Mar de Ojotsk, por un lado, y otra relativa al Océano Ártico, por el otro. La petición sobre el Mar de Ojotsk se recibió en la Comisión en el año 2013 y ha tenido por el momento una conclusión positiva; mientras que la petición revisada relativa al Océano Ártico se presentó en 2015 y sigue pendiente. Dinamarca ha presentado junto con Groenlandia dos reclamaciones. La primera, de junio de 2012, se refiere a la plataforma continental al sur de Groenlandia, un área que divide en una parte sur-occidental en el Mar de Labrador y una parte oriental del Mar Irminger. La segunda se presentó en noviembre de 2013 y se refería a la plataforma continental al noreste de Groenlandia, afectando el área entre Groenlandia y el Archipiélago de Svalbard. Noruega presentó en 2006 una petición en relación a tres áreas distintas: el Loop Hole en el mar de Barents, la cuenca occidental de Nansen en el Océano Ártico y el Banana Hole en el Mar de Noruega. La delimitación en el Mar de Barents, desde los años setenta objeto de discusión entre la ex Unión Soviética y Noruega, se sujeta ahora al Acuerdo entre Noruega y la Federación Rusa sobre la delimitación marina y la cooperación en el Mar de Barents y el Océano Ártico de 15 de setiembre de 2010. Finalmente, el gobierno canadiense también presentó en 2013 una demanda parcial ante la Comisión de Límites con carácter preliminar y que afecta propiamente a la ampliación de su plataforma continental a lo largo de la cordillera submarina Lomonosov, desde la isla de Ellesmere hasta Siberia, una reclamación que extiende la jurisdicción canadiense casi hasta el Polo Norte. Vid., sobre el estado de estas peticiones, Submissions, through the Secretary-General of the United Nations, to the Commission on the Limits of the Continental Shelf, pursuant to article 76, paragraph 8, of the United Nations Convention on the Law of the Sea of 10 December 1982, disponible online en http://www.un.org/depts/los/clcs_new/commission_submissions.htm. Para un comentario, Vid., MANERO SALVADOR, A., El deshielo del Ártico: Retos para el derecho internacional. La delimitación de espacios marinos y la protección y preservación del medio ambiente, Aranzadi-Thomson Reuters, 2011; CONDE PÉREZ, E., "Delimitaciones marítimas y territoriales en el Ártico: Desarrollo y tendencias", Revista Española de Derecho Internacional, Vol. 68 (1), 2016, pp. 235-239.
} 
de América del Norte, el campo de la Bahía de Prudhoe en Alaska, donde empezaron a extraer en 1977. Shell y BP iniciaron sus prospecciones mar adentro en el mar de Beaufort en los años ochenta, pero debido a las dificultades técnicas y los excesivos costes de producción acabaron abandonando estas explotaciones. En Canadá, la actividad extractiva se fue trasladando a partir de los años sesenta desde los Territorios del Noroeste hasta el Delta del Mackenzie, las Islas del Ártico y la Cuenca Sverdrup y a principios de los años setenta se iniciaron las primeras prospecciones mar adentro en el Mar de Beaufort canadiense. El desarrollo de esta industria en Groenlandia ha sido más complejo debido a la dificultad física que presenta este territorio y sus condiciones climáticas extremas. Aún así, las primeras prospecciones sísmicas y la perforación de pozos en su costa oeste se iniciaron en los años sesenta, mientras que en la costa este no empezó hasta 2011. La prospección sísmica del Mar de Barents noruego data de la década de los setenta, y la perforación mar adentro se lleva a cabo desde 1980. En Rusia el primer campo de gas mar adentro se inauguró en 1983 en el Mar de Barents, tres años antes del descubrimiento del primer campo de petróleo mar adentro en SeveroGulyaevskoe $^{11}$. Entre 2003 y 2008, cuando los precios del petróleo alcanzaron su máximo nivel, las principales compañías petroleras y gasísticas adquirieron mayoritariamente los arrendamientos de prospección y perforación en territorio ruso.

Ello no obstante, las actividades relacionadas con la extracción de petróleo y gas mar adentro en el Ártico siguen teniendo actualmente una importancia secundaria en el conjunto global ${ }^{12}$. En parte se debe a las dificultades técnicas que este tipo de operaciones presenta en un entorno tan especial. De modo general, la perforación mar adentro se está produciendo por ahora en aguas poco profundas, pero las mayores expectativas de la industria petrolera están puestas en un desarrollo tecnológico que facilite la perforación en aguas profundas como las del Ártico, siendo éste un aspecto condicionado aún por las consecuencias del vertido de petróleo en el Golfo de México en $2010^{13}$.

\footnotetext{
${ }^{11}$ Eurasia Group Report for the Wilson Center, Opportunities and Challenges for Arctic Oil and Gas Developement, The Wilson Center, Washington DC, 2014, pp. 4 y ss., disponible online en https://www.wilsoncenter.org/sites/default/files/Artic\%20Report_F2.pdf.

${ }^{12}$ LAJEUNESSE, A., "The New Economics of North American Arctic Oil", American review of Canadian Studies, Vol. 43 (1), 2013, pp. 107-122.

${ }^{13} \mathrm{El}$ vertido originado por un accidente en la plataforma de BP Deepwater Horizon se alargó durante 87 días,durante los que se derramaron cerca de 758 millones de litros de crudo al mar.Existe abundante bibliografía sobre este accidente. Entre otros: HOUCK, O.A., "Worst Case and the Deepwater Horizon Blowout: There Ought to Be a Law”, Tulane Environmental Law Journal, Vol. 24 (1), 2010, pp. 1-18;
} 
A ello se añade una ubicación extremadamente remota, con temperaturas de hasta $50^{\circ} \mathrm{C}$ bajo cero, una ecología cambiante, muy vulnerable y aun bastante desconocida que depende altamente de la condición del hielo marino, y unas características de luz muy particulares, en las que predominan los largos periodos de obscuridad. Un dato que indica claramente la dificultad de estas operaciones en el Ártico es el tiempo que transcurre entre la prospección y la primera extracción de petróleo, que de promedio requiere alrededor de veinte años ${ }^{14}$. Por todo ello la producción offshore en el Ártico resulta actualmente mucho más costosa que en la mayoría de las otras regiones gasísticas o petroleras del mundo. Las actividades de prospección y explotación sólo son rentables con una previsión de precios altos, además de exigir avances tecnológicos importantes en varias áreas, apoyo político (en particular en el régimen fiscal) y la amplia cooperación entre los Estados árticos para la prevención de los riesgos ambientales, especialmente en lo que se refiere a la preparación y respuesta ante posibles vertidos de petróleo ${ }^{15}$.

Las expectativas de desarrollo de este nuevo espacio están vinculadas a tres elementos particulares: el proceso de deshielo, el análisis coste/beneficio de la actividad de explotación de hidrocarburos y el desarrollo de tecnologías aplicables a la explotación de estos recursos en la región. El papel que tiene esta zona en la nueva configuración del espacio energético global también depende de la capacidad de los operadores de encontrar nuevas áreas de explotación y del aumento de las reservas mediante su identificación más efectiva. Hasta ahora, la recomposición del espacio geo-energético

NORCHI, Ch.H., "The Deepwater Horizon Incident", Ocean and Coastal Law Journal, Vol. 16 (2), 2011, pp. 245-252; OSOFSKY, H.M. "Multidimensional Governance and the BP Deepwater Horizon Oil Spill”, Florida Law Review, Vol. 63 (5), 2011, pp. 1077-1138; BRATSPIES, R.M., “A Regulatory Wakeup Call: Lessons from BP's Deepwater Horizon Disaster", Golden Gate University Environmental Law Journal, Vol. 5, 2011, pp. 7-60; SCHOENBAUM, Th.j., "Liability for Damages in Oil Spill Accidents: Evaluating the USA and International Law Regimes in the Light of Deepwater Horizon", Journal of Environmental Law, Vol. 24 (3), 2012, pp. 395-416; SCHOENBAUM, Th.J., "The Deepwater Horizon Oil Spill in the Context of the Public International Law Regimes for the Protection of the Marine Environment: A Comparative Study", University of San Francisco Maritime Law Journal, Vol. 25, 20122013, pp. 1-36; VINOGRADOV, S., "The Impact of the Deepwater Horizon: The Evolving International Legal Regime for Offshore Accidental Pollution Prevention, Preparedness, and Response", Ocean Development and International Law, Vol. 44 (4), 2013, pp. 335-362; SPICER, W., "Deepwater Horizon Lessons for the Offshore", Dalhousie Law Journal, Vol. 37 (1), 2014, pp. 205-232.

${ }^{14}$ DUESUND, J.M., Arctic Offshore gas \& Oil Outlook, Rystad Energy, 2015, disponible online en https://www.rystadenergy.com/NewsEvents/PressReleases/arctic-offshore-oil,-gas-outlook.

${ }^{15}$ HENDERSON, J., LOE, J., The Prospects and Challenges for Arctic Oil Development, OIES Paper: WPM 54, Oxford Institute for Energy Studies, 2014, p. 2. 
mundial ${ }^{16}$ y especialmente los cambios en el mercado mundial del petróleo y el gas inducidos por el desarrollo de nuevas tecnologías no convencionales de extracción se han traducido en un menor atractivo para la explotación de los hidrocarburos árticos. El impacto más significativo hasta el momento ha sido el de paralizar los proyectos de desarrollo mar adentro en el Ártico. Desde 2015 Shell y Statoil han ido cancelado los planes de prospección de petróleo en el Mar de Chukchi, mientras que ConocoPhillips, Chevron, BP y Exxon Mobile, aunque mantienen sus intereses en la región, también han ido ralentizando sus actividades en esta zona ${ }^{17}$. Asimismo, Repsol anunció en 2016 que también abandonaba sus planes de prospección en el Mar de Chukchi y en el Mar de Beaufort $^{18}$. De hecho, en 2016, la mayoría de compañías petrolíferas presentes en la zona habían renunciado a casi el $80 \%$ de los contratos de arrendamiento en el Mar de Chukchi, en medio de la caída general de los precios del petróleo ${ }^{19}$.

Aunque esta desaceleración en la puesta en marcha de los proyectos de explotación permite considerar más pausadamente los esfuerzos de regulación de esta actividad ${ }^{20}$, incluso si las dificultades del medio físico y la reducción de los precios del petróleo y del gas los retrasan algunos años y a menos que se lleve a cabo una auténtica transición en fuentes de energía, la demanda de petróleo y gas continuará creando presión para acceder a los recursos del Ártico ${ }^{21}$. Puesto que la explotación en las zonas más remotas requiere la movilización de múltiples buques durante un largo período de tiempo, cabe pensar también que un uso más intenso de las rutas navegables, por ahora concentradas

\footnotetext{
${ }^{16}$ Vid. BRIDGE, G., BOUZAROVSKI, S., BRADSHAW, M., \& EYRE, N., "Geographies of energy transition: Space, place and the low-carbon economy", Energy Policy, Vol. 53, 2013, pp. 331-340; MAÑÉ ESTRADA, A., (2006). "European energy security: Towards the creation of the geo-energy space", Energy Policy, Vol. 34, n 18, pp. 3773-3786.

${ }^{17}$ HOAG, H., "Arctic Development Stalls with Tumbling Oil Prices", Arctic Deeply, enero 2016, disponible online en https://www.newsdeeply.com/arctic/articles/2016/01/22/arctic-development-stallswith-tumbling-oilprices; DLOUHY, J.A., "Big Oil Abandons \$2.5 Billion in U.S. Arctic Drilling Rights", Bloomberg, mayo 2016, disponible online en https://www.bloomberg.com/news/articles/2016-05-10/bigoil-abandons-2-5-billion-in-u-s-arctic-drilling-rights.
}

${ }^{18}$ ESTELLER, R, "Repsol renuncia a cien yacimientos en Alaska, Namibia, Liberia y Nicaragua", ElEconomista.es, junio 2016, disponible online en http://www.eleconomista.es/empresasfinanzas/noticias/7626526/06/16/Repsol-renuncia-a-cien-yacimientos-en-Alaska-Namibia-Liberia-yNicaragua.html.

${ }^{19}$ Arctic Law \& Policy Institute, "Arctic Law \& Policy Year in review 2016", Washington Journal of Environmental Law \& Policy, Vol. 7, 2017, pp. 117-250, p. 123.

${ }^{20}$ Vid., GULAS, S., DOWNTON, M., D'SOUZA, K., HAYDEN, K., WALKER, T.R., "Declining Arctic Ocean oil and gas developments: Opportunities to improve and environmental pollution control", Marine Policy, Vol. 75, 2017, pp. 53-61.

${ }^{21}$ OSOFSKY, H.M., SHADIAN, J., FECHTELKOTTER, S.L., “Arctic Energy Cooperation”,U.C. Davis Law Review, Vol. 49,2016, pp. 1431-1510, pp. 1440 y ss. 
en las navegaciones en la periferia del Círculo Ártico, facilitará esta explotación gracias a un ahorro significativo en tiempo y combustible en su transporte. De hecho, en Canadá y en Estados Unidos siguen planificándose, aunque a un paso más lento, nuevas exploraciones en el Ártico ${ }^{22}$. Asimismo, las compañías noruegas mantienen sus proyectos en el Mar de Barents, si bien ahora bajo una fuerte presión de las organizaciones ambientales ${ }^{23}$ y el gigante ruso Gazprom continua explotando los hidrocarburos en el Mar de Pechora. En particular, la Federación Rusa sigue proporcionando por ahora el mayor número de arrendamientos para la explotación de hidrocarburos árticos mediante proyectos conjuntos en los que Rosneft participa junto con compañías extranjeras como Exxon Mobil, Statoil y Eni (si bien estos contratos se han reducido mucho y en su mayoría se firmaron antes de que la producción de esquisto se generalizara) o las asiáticas Inpex y $\mathrm{ONGC}^{24}$. Por tanto, cabe esperar que aunque no se produzca una actividad significativa a corto o medio plazo ésta aumente a largo plazo, en un escenario en el que se mantenga la dependencia de los combustibles fósiles y empiece a decaer la fiebre por otras formas de extracción no convencionales (el volumen de cuyas reservas se ha sobreestimado, son también finitas y cada vez más están sujetas a mayores obstáculos legales debido a sus consecuencias ambientales) ${ }^{25}$.

\section{La realidad del riesgo ambiental: Implicaciones de una contaminación por hidrocarburos en el Ártico}

Las consecuencias ambientales de la exploración y la explotación mar adentro de hidrocarburos son inevitables y se manifiestan de múltiples formas. Entre ellas cabe señalar la producción de sonidos ultramarinos debido a las operaciones sísmicas, la

${ }^{22}$ World Oil, 2015, disponible online en http://www.worldoil.com/news/2015/9/21/hilcorp-submitsdevelopment-plan-for- liberty-prospect-offshore-alaska.

${ }^{23}$ El pasado 18 de octubre 2016, las organizaciones ambientalistas Foreningen Greenpeace Norden y Natur og Ungdom introdujeron una demanda ante la Corte de Distrito de Oslo contra el gobierno noruego, reclamando la anulación del procedimiento de concesión de licencias para la prospección petrolífera en el Mar de Barents por vulnerar los compromisos asumidos en el Acuerdo de París y el artículo 112 de la Constitución noruega. Vid. Office of the General prosecutor, Foreningen Greenpeace Norden and Natur og Undgdom v. The Government of Norway (Ministry of Petroleum and Energy), case ${ }^{\circ}{ }^{\circ} 16-166674 T V I-$ OTIR/06, traducción no oficial al inglés disponible online en https://www.savethearctic.org/en/peoplevsarcticoil/blog/background-documents/

${ }^{24}$ Vid. HENDERSON, J., LOE, J., The Prospects and Challenges for Arctic Oil Development, ob.cit., p. 2.; DUESUND, J.M., Arctic Offshore gas \& Oil Outlook, ob.cit.

${ }^{25}$ Vid. al respecto, ESCRIBANO, G., "EE UU como poder energético no convencional y sus limitaciones" y ROGERS, D., "Gas y petróleo de esquisto, una falsa seguridad", Economia Exterior, Vol. 68, 2014, pp. 9-18 y 69-78, respectivamente. 
presencia de desechos procedentes de las perforaciones y de mayores cantidades de plásticos en el mar, la creciente contaminación por contaminantes orgánicos persistentes, metales pesados y otros contaminantes por las descargas asociadas a las operaciones de perforación y la contaminación por hidrocarburos por fugas menores y filtraciones durante las operaciones de prospección y producción ${ }^{26}$.

En condiciones normales, por tanto, los retos ambientales a los que se enfrenta la región ártica por la presencia de infraestructuras asociadas a esta actividad son muy significativos ${ }^{27}$. Los sonidos submarinos de las plataformas de prospección y perforación, de los buques o de la simple rotura del hielo pueden dañar o interferir en el comportamiento vital de mamíferos marinos, peces e invertebrados. Las prospecciones sísmicas también afectan a las pesquerías de importancia comercial, la reducción de cuyas tasas de captura puede persistir mucho después de la terminación de este tipo de operaciones. En el Ártico, además, la pérdida de hielo marino debido al carbono negro, incluyendo el emitido por las operaciones petroleras, reduce el hábitat esencial de las especies autóctonas. A medida que aumenta la temperatura en el océano y en la atmosfera se produce un desplazamiento de las especies del hemisferio norte hacia el Polo Norte, algo que obviamente resulta más limitado para los mamíferos marinos del Ártico. Asimismo, los hábitats primarios de muchas de estas especies se encuentran en la plataforma continental, con lo que este desplazamiento solo puede realizarse hacia el Océano Ártico Central, más profundo y menos productivo para su supervivencia. Aquellas especies que no se desplazan se enfrentan al calentamiento del medio marino y al retroceso del hielo, que modifica las condiciones necesarias para satisfacer sus necesidades alimenticias, reproductivas o, simplemente, térmicas.

A su vez, la explotación de hidrocarburos contribuye con cantidades significativas de emisiones de gases al efecto invernadero que, a su vez, conduce a un mayor calentamiento y acidificación del medio marino ${ }^{28}$. Tan solo la explotación de las

\footnotetext{
${ }^{26}$ Arctic Monitoring and Assessment Programme, Arctic Oil and Gas 2007, 2008, disponible online en https://www.amap.no/documents/doc/arctic-oil-and-gas-2007/71.

${ }^{27}$ Para un estudio detallado, ver el informe elaborado por U.S. Department of Commerce -National Oceanic and Atmospheric Administration -National Marine Fisheries Service -Office of Protected Resources, Effects of Oil and Gas Activities in the Arctic Ocean.Final Environmental Impact Statement, Octubre 2016, disponible en http://www.nmfs.noaa.gov/pr/permits/eis/volume_2_arctic_seismic_feis_oct_2016_final_publication_ver sion.pdf.

${ }^{28}$ Arctic Monitoring and Assessment Programme, Arctic Ocean Acidification 2013: An Overview, disponible en http://www.amap.no/documents/doc/arctic-ocean-acidification-2013-an-overview/1061.
} 
reservas de petróleo y gas en el Océano Ártico bajo jurisdicción de Estados Unidos puede liberar hasta 15.800 millones de toneladas de dióxido de carbono a la atmósfera, aproximadamente el equivalente a las emisiones de todos los modos de transporte estadounidenses en un período de nueve años. Esto genera un efecto perverso: el fenómeno del cambio climático, una de cuyas consecuencias principales es la disminución de la extensión de las zonas heladas en el Ártico, está facilitando la perforación mar adentro de yacimientos de gas y petróleo que generan, a su vez, un aumento de la combustión de combustibles fósiles y de emisiones de gases de efecto invernadero resultantes. Todo ello resulta en mayores presiones ambientales sobre el territorio, en un espacio cuya integridad está ya altamente comprometida por la erosión costera, el deshielo del permafrost y las condiciones meteorológicas extremas.

Sin embargo, el riesgo más importante reside en los vertidos masivos de petróleo debidos a accidentes en los pozos petrolíferos de las plataformas mar adentro, que en esta región pueden dañar irreparablemente el ecosistema marino y su fauna. Hasta ahora el más importante ha sido el de la plataforma Deepwater Horizon en el Golfo de México en 2010. Aunque en los últimos años el registro de vertidos desde plataformas mar adentro en el Ártico ha mostrado una relativa mejora ${ }^{29}$-incluso el National Research Council declaró en 2003 la improbabilidad de que pudieran producirse este tipo de vertidos en el norte de Alaska ${ }^{30}$-, las agencias federales también llegaron a una conclusión similar respecto a la perforación en aguas del Golfo de México antes de que se produjera el accidente del Deepwater Horizon $^{31}$.

Evidentemente, ambas situaciones difieren por las características de la zona geográfica y por las condiciones en que se producen las actividades de exploración y explotación (especialmente en lo que se refiere a la profundidad de los pozos y la presión del agua), pero no puede ignorarse que incluso bajo los sistemas de control más rigurosos este tipo de vertido puede volver a producirse por estimaciones erróneas, un fallo técnico o simplemente un error humano. La respuesta al accidente y posterior vertido del

\footnotetext{
${ }^{29}$ National Research Council, Oil in the Sea III: Inputs, Fates, and Effects,2003 y Oil Spills: Background and Governance, Analysis of U.S. Oil Spillage, (Prepared for American Petroleum Institute), Report RL33705, 2009.

${ }^{30}$ National Research Council, Cumulative Environmental Effects of Oil and Gas Activities on Alaska's North Slope, 2003.

${ }^{31}$ US Department of Interior Minerals Management Service, Outer Continental Shelf Oil \& Gas Leasing Program: 2007-2012, Final Environmental Impact Statement, 2007, Chapter 4.
} 
Deepwater Horizon ejemplifica claramente la complejidad técnica, jurídica y económica, además del coste ambiental y humano, que plantearía una situación similar en las aguas del Ártico.

En términos generales, los desafíos logísticos y tecnológicos que conllevan estas actividades constituyen el factor principal que incrementa el riesgo de contaminación en el Ártico ${ }^{32}$. Por ahora no hay una tecnología absolutamente fiable para hacer frente a estos vertidos y los métodos tradicionales de respuesta parecen ser ineficaces en el Ártico. Por otro lado, es obvio que ningún accidente de este tipo es totalmente benigno o irrelevante y todos tienen un mayor o menor impacto en el medio. Incluso un pequeño vertido de petróleo en el mar, dependiendo del momento y el lugar, puede causar un daño significativo en los ecosistemas y afectar a las poblaciones de mamíferos marinos, los peces, las aves, o las especies que viven en el fondo marino. En el caso del Océano Ártico, estos vertidos pueden tener implicaciones extremadamente graves y aun poco conocidas. Las condiciones en la región imponen desafíos únicos en lo que concierne a la capacidad de respuesta, las operaciones de limpieza y la recuperación del petróleo así como en lo que se refiere a los mecanismos para mitigar la contaminación y el daño al ecosistema, puesto que su comportamiento en aguas heladas no se conoce tan bien como el que se produce en climas más templados ${ }^{33}$.

\footnotetext{
${ }^{32} \mathrm{El}$ desarrollo de estas actividades, y especialmente las consecuencias ambientales que conllevan en un entorno tan especial, han constituido una preocupación constante de la comunidad internacional. A pesar de ello, el marco jurídico internacional existente relativo a los riesgos que presentan las operaciones marítimas en la región del Ártico sigue siendo incompleta y fragmentada, además de que frecuentemente la aplicación de los anexos a estos tratados tiene un carácter voluntario. Entre los acuerdos adoptados en el seno de la OMI que inciden la navegación por aguas polares cabe mencionar aquí el Convenio OPRC de 30 de noviembre de 1990 sobre cooperación, preparación y lucha contra la contaminación por hidrocarburos (BOE 5 de junio de 1995), del que son parte todos los Estados ribereños del Ártico; el Convenio MARPOL 73/78 relativo a la prevención de la contaminación ocasionada por los buques (BOE 17-18 de octubre de 1984) y, más recientemente, adoptado el 15 de mayo de 2015 mediante la última enmienda del Convenio MARPOL, el Código Internacional para buques que naveguen en aguas polares (Código Polar) adoptado en 2015 (RESOLUCIÓN MEPC.264(68) (BOE 5 de mayo de 2017), en vigor desde 2017, que afecta a la construcción, equipo, funcionamiento, búsqueda y salvamento y protección del medio marino en los casquetes polares. Entre los acuerdos elaborados en el seno del Consejo Ártico es especialmente relevante el Acuerdo de Cooperación para la prevención y respuesta de la contaminación marina procedente de vertidos de hidrocarburos en el Ártico adoptado en la Conferencia Ministeral de Kiruna el 15 de mayo de 2013, en vigor desde 2016 (texto disponible online en http://arcticcouncil.org/eppr/wp-content/uploads/2016/06/EDOCS-2067-v1-

ACMMSE08_KIRUNA_2013_agreement_on_oil_pollution_preparedness_and_response_in_the_arctic_ formatted.pdf).

${ }^{33}$ National Research Council, Responding to Oil Spills in the U.S. Arctic Marine Environment, 2014; Vid.HULTS, D., "Environmental Regulation at the Frontier: Government Oversight of Offshore Oil Drilling North of Alaska", Environmental Law, Vol. 44, 2014, pp. 761-832, pp. 808-816.
} 
Su impacto puede variar también en el tiempo y sus consecuencias permanecer desde unos pocos días hasta varios años, incluso décadas en algunos $\operatorname{casos}^{34}$. Los vertidos de petróleo en el permafrost pueden persistir en los ecosistemas durante periodos de tiempo aún más largos, dañando potencialmente la fauna y la flora ártica. De acuerdo con el National Research Council, en temperaturas más frías o en el hielo marino los procesos que controlan el comportamiento del petróleo, tales como la difusión, la evaporación, la foto-oxidación, la emulsificación, o la dispersión natural pueden modificarse por períodos prolongados de tiempo ${ }^{35}$. Aunque al darse un proceso más lento de meteorización el tiempo para implementar estrategias de respuesta también puede ser mayor, no puede ignorarse que cuanto más tiempo permanece el petróleo en un ecosistema mayor es la exposición y por ahora se conoce poco sobre la capacidad de especies únicas en el Ártico para sobrevivir o recuperarse en este tipo de situaciones ${ }^{36}$.

Relacionado con este aspecto, el tiempo de respuesta es un factor crítico para hacer frente a los vertidos de petróleo. Con cada hora, el petróleo derramado se vuelve más difícil de rastrear, contener y recuperar, sobre todo en condiciones de hielo en las que el vertido puede desplazarse debajo del mismo o mezclarse con la zona helada. La mayoría de las técnicas de respuesta requieren una acción rápida, lo que plantea importantes problemas logísticos en zonas en las que no se han realizado acciones previas, faltan infraestructuras o no hay profesionales capacitados para abordar las acciones de respuesta. El informe de la US Government Accountability Office identificó en 2010 diversos obstáculos de esta naturaleza que impedían una respuesta adecuada y eficaz a los vertidos de petróleo en la región, incluyendo una información deficiente o inadecuada sobre el Océano Ártico y los problemas tecnológicos relativos a las comunicaciones ${ }^{37}$.

La imprevisibilidad del hielo ártico y de los témpanos de hielo constituye otro de los obstáculos para hacer frente a este tipo de situaciones. Mientras que los bloques de hielo permanente pueden tener más de tres metros de espesor, los bloques de menor espesor y

\footnotetext{
${ }^{34}$ Arctic Council, Guide to Oil Spill Response in Snow and Ice Conditions, Emergency Prevention, Preparedness and Response Working Group 2015, disponible online en https://oaarchive.arcticcouncil.org/handle/11374/403.

${ }^{35}$ National Research Council, Responding to Oil Spills in the U.S. Arctic Marine Environment, ob.cit.

${ }^{36}$ Arctic Monitoring and Assessment Programme, Arctic Oil and Gas 2007, ob.cit.

${ }^{37}$ US Government Accountability Office, Coast Guard: Efforts to Identify Arctic Requirements Are Ongoing, but More Communication about Agency Planning Efforts Would Be Beneficial, GAO-10-870, September 2010.
} 
antigüedad pueden ser partidos por los rompehielos tradicionales o incluso por buques aptos para este tipo de navegación. En el Paso del Noreste en especial, la fusión del hielo y la apertura de aguas que en su momento estaban cubiertas con capas de hielo de un año ha permitido la llegada o el surgimiento de bloques de hielo permanente y de icebergs procedentes de Groenlandia, que ahora transitan dentro de las líneas comerciales de navegación y se acercan también a las plataformas mar adentro.

Otro factor a considerar es el de la falta de infraestructuras en la región ártica, una carencia reconocida por el informe elaborado por el National Research Council en $2014^{38}$. La Coast Guard de Estados Unidos, la más preparada en la región, no tiene instalaciones terrestres apropiadas para este fin más al norte de Kodiak, en Alaska, a $1.600 \mathrm{~km}$. del territorio más septentrional de la costa de Alaska. Aunque algunas de las poblaciones en esta zona tienen pistas de aterrizaje para vuelos de carga no cuentan con carreteras que conecten estas infraestructuras. Las instalaciones portuarias son también muy deficientes, con una ausencia considerable de infraestructuras ship-to-shore y estaciones de abastecimiento y con el puerto más cercano, el de las Islas Aleutianas, a más de $2000 \mathrm{~km}$. de Point Barrow ${ }^{39}$. Es además necesario realizar una importante inversión en infraestructuras marítimas para asegurar el transporte trans-ártico sin un riesgo excesivo. La utilización de las tradicionales señales marinas tipo boyas u otros mecanismos visuales no son viables en el Ártico porque el movimiento constante del hielo modifica sus posiciones. Por esta razón, la navegación está limitada al uso de las cartas marinas, que en su mayoría están incompletas o anticuadas. Para remediar este problema, los operadores necesitan acceder a los reconocimientos aéreos del estado del hielo y a las imágenes por satélite. Existe pues una laguna importante respecto a las acciones a emprender ante un vertido de petróleo en esta región ${ }^{40}$, que implica una situación de inseguridad que, comparada con lo que puede ocurrir en otras zonas del planeta, puede ser mucho mayor y agrava considerablemente el riesgo de consecuencias irreversibles en el Ártico.

\footnotetext{
${ }^{38}$ National Research Council, Responding to Oil Spills in the U.S. Arctic Marine Environment, ob.cit.

${ }^{39}$ U.S. Coast Guard, Report to Congress: U.S. Coast Guard Polar Operations, 2008; Nuka Research and Planning Group, Oil Spill Prevention and Response in the U.S. Arctic Ocean: Unexamined Risks, Unacceptable Consequences, Pew Environment Group, 2010; World Wildlife Fund, Oil Spill: Response Challenges in Arctic Waters, 2007; National Research Council, Oil Spills: Background and Governance, Analysis of U.S. Oil Spillage, ob.cit.

${ }^{40}$ Coastal Response Research Center, Opening the Arctic Seas: Envisioning Disasters and Framing Solutions, 2009.
} 


\section{LOS INTERESES ESTRATÉGICOS DE ESTADOS UNIDOS EN EL ÁRTICO Y SU POLÍTICA EN RELACIÓN A LA EXPLORACIÓN Y EXPLOTACIÓN DE SUS HIDROCARBUROS}

Los intereses de Estados Unidos y otros Estados ribereños, no ribereños e incluso no árticos ${ }^{41}$ en la región difieren de manera significativa en lo que se refiere a sus estratégicas árticas, diferencias que son más matizadas con respecto a la exploración y explotación de los hidrocarburos árticos ${ }^{42}$. Estados Unidos ha concedido tradicionalmente una relativa importancia al Ártico ${ }^{43}$, por lo que con frecuencia ha sido calificado como un reluctant Arctic power por su política en exceso reactiva, rígida y poco sistemática ${ }^{44}$. Entre los restantes Estados ribereños, Canadá ${ }^{45}$ y Groenlandia (Dinamarca) $)^{46}$ tienen un interés relativo en la explotación de estos recursos por motivos

\footnotetext{
${ }^{41}$ La presencia en la región de los Estados árticos no ribereños y los Estados no árticos de estos Estados se canaliza esencialmente a través del Consejo Ártico, a la que pertenecen con el estatuto de miembros, por su carácter de Estados árticos no ribereños, Finlandia y Suecia e Islandia. También participan en el Consejo Ártico, con el estatuto de observadores permanentes, otros actores geográficamente ajenos a la región que en un futuro pueden influir claramente en el proceso de gobernanza ambiental del Ártico: Desde 1998 tienen este estatuto Alemania, los Países Bajos, Polonia, Reino Unido; desde 2000 Francia; España desde 2006; desde 2013 Italia, China, India, Japón, Corea del Sur y Singapur y, desde 2017, Suiza. La Unión Europea sigue teniendo por ahora un estatuto de observador ad hoc.
}

${ }^{42}$ KEIL, K. "Spreading Oil, Spreading Conflict? Institutions Regulating Arctic Oil and Gas Activities", The International Spectator, Vol. 50 (1), 2015, pp. 85-110, pp. 87 y ss. y 92 y ss.; GULAS, S., DOWNTON, M., D'SOUZA, K., HAYDEN, K., WALKER, T.R., "Declining Arctic Ocean oil and gas developments: Opportunities to improve and environmental pollution control”, ob.cit., pp. 57-58.

${ }^{43}$ CONLEY, H., KRAUT, J., U.S. Strategic Interests in the Arctic. An Assessment of Current Challenges and New Opportunities for Cooperation, CSIS Europe Program, Center for Strategic \& International Studies, 2010, disponible $\quad$ online en http://csis.org/files/publication/100426_Conley_USStrategicInterests_Web.pdf.

${ }^{44}$ HUEBERT, R. The United States Arctic Policy: The Reluctant Arctic Power, The School of Public Policy, University of Calgary, SPP Briefing Papers Focus on the United States. Vo. 2 (2), 2009, pp.1-26 disponible online en http://policyschool.ucalgary.ca/files/publicpolicy/SPPBriefingHUEBERTonline.pdf.

${ }^{45}$ En Canadá el Ártico es prioritario en la agenda política desde 2006, con llegada al poder del partido conservador. Este Estado ha promovido tradicionalmente el reforzamiento de la soberanía nacional mediante una mayor presencia en la zona, especialmente en lo que se refiere a la reclamación de las aguas navegables. La estrategia del Gran Norte adoptada en 2009 por el gobierno canadiense (Government of Canada, Canada's Northern Strategy: Our North, Our Heritage, Our Future, 2009, disponible online en http://www.northernstrategy.ca/index-eng.asp) se ha revitalizado con la llegada del Primer Ministro Trudeau. La protección ambiental del Ártico y de sus comunidades y el desarrollo sostenible de la región han pasado a constituir el núcleo de la acción de Canadá, en una nueva perspectiva que se manifiesta en 2016 con el establecimiento de una nueva asociación con Estados Unidos (U.S.-Canada Joint Statement on Climate, Energy, and Arctic Leadership, 10.3.2016, disponble online en http://pm.gc.ca/eng/news/2016/03/10/us-canada-joint-statement-climate-energy-and-arctic-leadership).

${ }^{46}$ El único Estado miembro de la Unión Europea en formar parte de este grupo es Dinamarca, que cuenta desde 2016 con una nueva estrategia para el Ártico (Strategi for forskning og uddannelse vedrørende 
diferentes. Mientras que para Canadá, el gas y el petróleo del Ártico son importantes principalmente por lo que suponen de ejercicio de la soberanía sobre el territorio, el principal interés de Groenlandia (Dinamarca) está vinculado con la ambición de una mayor autonomía política y económica. En cambio, para la Federación Rusa ${ }^{47}$ y para Noruega ${ }^{48}$ los hidrocarburos del Ártico tienen una enorme importancia debido a una combinación de razones que afectan a la seguridad, el desarrollo económico y la identidad nacional ${ }^{49}$.

\section{La posición de Estados Unidos en el Ártico}

De acuerdo con los datos del USGS ${ }^{50}$, el depósito de petróleo situado en la zona ártica de Alaska convierte a Estados Unidos en el segundo productor de petróleo del Ártico.

Arktis, Uddannelses- og Forskningsministeriet, 2016, disponible online en
https://ufm.dk/publikationer/2016/filer/fi_rapport_arktisk_strategi_web.pdf). Su condición como Estado
ribereño del Ártico se deriva de su vinculación con Groenlandia, que posee un estatuto de autonomía
propio desde 1979. Aunque Groenlandia no tiene aun un desarrollo notable de la industria del gas y
petróleo, el informe de 2008 del USGS le atribuye un volumen potencial de hidrocarburos cercano al 10\%
del total estimado de las reservas de petróleo y gas ártico. Además, el este de Groenlandia está clasificado
entre las zonas petrolíferas potencialmente más grandes del mundo.

${ }^{47}$ La Federación Rusa constituye hoy en día el más importante actor en el Ártico, no solo por la longitud de su costa ártica sino también por la actividad que ha llevado a cabo en ella históricamente, que se remonta a la primera década del siglo XX. La región del Ártico, sus hidrocarburos y la apertura de las nuevas rutas de navegación son un motor clave para el desarrollo económico del país en el siglo XXI. De acuerdo con el USGS, el Ártico ruso concentra el 52\% del total del petróleo estimado en la región, además de unas reservas de gas que corresponden al 23,9\% del total del mundo. El carácter estratégico que ha tenido siempre el Ártico para la Federación Rusa se expresa claramente en la estrategia oficial adoptada en 2008 (Security Council of Russia, Principles of State Policy in the Arctic to 2020, 18 September 2008, disponible online en http://www.scrf.gov.ru/documents/98.html). Sin embargo, la actividad económica de este país en el Ártico se ha reducido en los últimos años. Por un lado, el desarrollo de estos depósitos de hidrocarburos requiere el uso de tecnologías de perforación en aguas profundas de las que actualmente la Federación Rusa no dispone, de modo que su uso en la plataforma continental es difícil o incluso imposible de realizar sin la participación de socios y capital extranjero. Por otro lado, las sanciones económicas a raíz de la crisis de Ucrania han pospuesto algunas de las operaciones de exploración de yacimientos de gas natural y petróleo, con lo que empresas extranjeras que participan en proyectos energéticos en el Ártico ruso se están desplazando cada vez más hacia Asia y China.

${ }^{48}$ Noruega ha sido tradicionalmente el Estado más implicado en la gestión de esta zona, ya que tiene importantes lazos históricos, económicos y culturales con ella que incluso inciden en la formación de su identidad nacional. Por esta razón, Noruega ha sido muy activa en sus esfuerzos para transformar sus regiones del norte en centros relevantes de actividad económica y en particular, ha insistido en la utilización del Ártico y sus recursos estratégicos como base del crecimiento económico y del desarrollo nacionaly ha situado el Ártico como un elemento importante de la política exterior noruega (The Norwegian Government's High North Strategy, adoptado en el 2006 por el Ministerio de Asuntos Exteriores, disponible online en http://www.regjeringen.no/upload/UD/vedlegg/strategien.pdf).

${ }^{49}$ KEIL, K., "The Arctic: A new region of conflict? The case of oil and gas", Cooperation and Conflict 2014, Vol. 49 (2), 2014, pp. 162-190.

${ }^{50}$ BIRD, K.J et al., Circum-Arctic Resource Appraisal: Estimates of Undiscovered Oil and Gas North of the Arctic Circle, ob.cit.; BUDZIK, Ph., Arctic Oil and Natural Gas Potential, U.S. Department of Energy, Energy Information Administration, 2009, disponible online en 
Las estimaciones realizadas hasta ahora apuntan a que la plataforma continental adyacente al territorio de Alaska contiene unos recursos de 27.000 millones de barriles de petróleo y 3.7 billones de $\mathrm{m}^{3}$ de gas natural, que en los próximos treinta y cinco años podrían contribuir a mantener los suministros internos cuando la producción de petróleo de esquisto bituminoso de Estados Unidos empezara a declinar ${ }^{51}$.

Sin embargo, este país, que concentra sus principales áreas de extracción de hidrocarburos en Texas y en el Golfo de México, ha dedicado hasta ahora una atención limitada al Ártico en general y a los hidrocarburos de la región en particular. En los años ochenta, su interés se limitaba a una posibilidad reducida de extracción de hidrocarburos en Alaska, y aun así, las expectativas no eran muy esperanzadoras debido a las dificultades físicas y técnicas ${ }^{52}$. El siglo XXI trajo consigo importantes cambios en el mercado mundial del petróleo y del gas. El desarrollo de nuevas tecnologías de extracción no convencionales ha facilitado el acceso a reservas de petróleo y gas que antes eran inaccesibles, lo que ha permitido un incremento de la producción de gas natural en un $35 \%$ entre 2005 y 2013, con una previsión de incrementarlo hasta un $45 \%$ en $2040^{53}$. Como observa el informe de la Brookings Energy Security Initiative de 2014, el éxito de la revolución de petróleo y gas no convencional ha tenido un efecto moderador sobre la rapidez con la que se han desarrollado las operaciones de extracción de recursos energéticos en zonas de alto coste, como el Ártico ${ }^{54}$. Aun con ello, las nuevas condiciones ambientales del Ártico y el interés comercial que ha despertado la explotación de sus recursos en una perspectiva a largo plazo siguen constituyendo un elemento clave de la estrategia ártica de Estados Unidos, si bien por ahora modulado

https://www.eia.gov/analysis/studies/archive/2009/arctic/pdf/arctic_oil.pdf.

${ }^{51}$ De acuerdo con las estimaciones del US Bureau of Ocean Energy Management (2017-2022 Outer Continental Shelf Oil and Gas Leasing Draft Proposed Program, 2015, disponible online en https://www.boem.gov/2017-2022-DPP/) y del National Petroleum Council (Arctic Potential: Realizing the Promise of U.S. Arctic Oil and Gas Resources, 2015, disponible online en http://www.npc.org/reports/research.html.), citados en O'ROURKE, R., Changes in the Arctic: Background and Issues for the Congress, Congressional Researchs Service, 2017, p. 27, disponible online en http://fas.org/sgp/crs/misc/R41153.pdf.

${ }_{52}$ Advisory Panel, Oil and Gas Technologies for- the Arctic and Deepwater, U.S. Congress, Office of Technology Assessment, OTA-O-270, 1985, pp. 5 y 7.

${ }^{53}$ U.S. Energy Information Administration, Annual Energy Outlook 2015 with Projections to 2040, 2015, pp. 1 y 21, disponible online en http://www.eia.gov/forecasts/aeo/pdf/0383(2015).pdf.

${ }^{54}$ EBINGER, CH., BANKS, J.P., SCHACKMANN, A., Offshore Oil and Gas Governance in the Arctic. A Leadership Role for the U.S., Brookings Institute, 2014, disponible online en https://www.brookings.edu/wp-content/uploads/2016/02/Offshore-Oil-and-Gas-Governance-web.pdf. 
por la entrada en escena de las fuentes no convencionales, junto con la crisis económica y los bajos precios del petróleo en el mercado mundial.

Al respecto pueden identificarse al menos tres periodos claros en la estrategia de Estados Unidos en torno a la región ártica marcados siempre por los intereses de la seguridad nacional.

El primero de los documentos estratégicos relacionados con el Ártico estadounidense se adoptó en 1971, bajo la presidencia del presidente Nixon. De tan solo dos páginas de extensión, la National Security Decision Memorandum (NSDM-144) sobre el Ártico ${ }^{55}$ se centraba en la protección de los intereses esenciales de la seguridad de Estados Unidos, incluyendo la preservación del principio de la libertad de los mares y el espacio aéreo. Posteriormente, ya bajo el mandato del presidente Reagan, la administración norteamericana adoptó en 1984 la Arctic Research and Policy Act (ARPA) ${ }^{56}$, con un contenido material limitado a la articulación de un "comprehensive national policy dealing with national research needs and objectives in the Arctic". Esta norma completaba parcialmente la brevísima referencia que en relación al Ártico hacia la National Security Decision Directive (NSDD-90) de $1983^{57}$ reafirmando los intereses críticos de Estados Unidos en relación con la defensa nacional, los recursos energéticos, la investigación científica y la protección del medio ambiente.

En segundo periodo se inicia en 1994 y se caracteriza por un emergente interés en la región que se refleja en dos nuevos documentos estratégicos. Así, ya bajo la administración del presidente Clinton se adopta la Presidential Decision Directive(PDD/NSC-26) on Arctic and Antarctic Policy ${ }^{58}$, que define por primera vez los objetivos concretos del gobierno estadounidense: “(1) meeting post-Cold War national security and defense needs, (2) protecting the Arctic environment and conserving its biological resources, (3) assuring that natural resource management and economic development in the region are environmentally sustainable, (4) strengthening institutions for cooperation among the eight Arctic nations, (5) involving the Arctic's indigenous peoples in decisions that affect them, and(6) enhancing

55 US National Security Council, National Security Decision Memorandum 144, Washington DC, December 22, 1971, disponible online https://fas.org/irp/offdocs/nsdm-nixon/nsdm-144.pdf

56 Title I of P.L. 98-373 de 31.7.1984. La ley es modificada por la P.L. 101-609 de 16.11.1990.

57 The White House, National Security Decision Directive (NSDD-90), United States Arctic Policy, 14.4.1983, disponible online en http://www.fas.org/irp/offdocs/nsdd/nsdd-090.htm.

58 The White House, Presidential Decision Directive/NSC-26, 9.6.1994, disponible online https://fas.org/irp/offdocs/pdd/pdd-26.pdf 
scientific monitoring and research into local, regional and global environmental issues." A pesar de que las tensiones de la Guerra Fría habían disminuido y se abrían nuevas posibilidades de cooperación con la ex Unión Soviética, los intereses estadounidenses seguían vinculándose directamente con la seguridad nacional y la defensa.

Al final de su mandato, la administración de George W. Bush adoptó el 9 de enero de 2009 la Presidential Directive NSPD-66/HSPD-25 $5^{59}$, que reemplazaba la de 1994 pero seguía en una línea muy similar. Además de presentar a Estados Unidos como una nación ártica con múltiples intereses en la región relacionados con la seguridad nacional, introduce diversos elementos como la gobernanza internacional, la cuestión de la extensión de la plataforma continental y los temas fronterizos, la promoción de la cooperación internacional en materia de investigación ártica y la política de transporte, energía y protección ambiental. Sin embargo y a diferencia de otros Estados árticos, en este periodo Estados Unidos seguía careciendo de una estrategia que priorizara el desarrollo y la protección de la región. Este elemento se contempla tan solo muy brevemente en la National Security Strategy, conforme al cual "The United States is an Arctic Nation with broad and fundamental interests in the Arctic region, where we seek to meet our national security needs, protect the environment, responsibly manage resources, account for indigenous communities, support scientific research, and strengthen international cooperation on a wide range of issues" ${ }^{60}$. A pesar de los cambios tanto en el paisaje geopolítico (el final de la Guerra Fría), como el físico (la reducción acelerada de la capa de hielo polar) no se producen cambios significativos en la posición estadounidense ${ }^{61}$.

La última etapa comprende desde 2010 hasta la actualidad y se significa por la adopción de una estrategia nacional específica para el Ártico por parte de la administración de Barak Obama el 10 de mayo de 2013 (National Strategy for the Arctic Region) ${ }^{62}$, que

\footnotetext{
59 The White House, National Security Presidential Directive 66/Homeland Security Presidential Directive 25 (NSPD 66/HSPD 25), disponible online en https://www.hsdl.org/?view\&did=232474

60 The White House, National Security Strategy, 2010, p. 50, disponible on line https://www.whitehouse.gov/sites/default/files/rss_viewer/national_security_strategy.pdf.

${ }^{61}$ CONLEY, H., The New Foreign Policy Frontier: US interests and actors in the Arctic, Center for Strategic and International Studies (CSIC), 2013, p. 3, disponible en https://narfu.ru/upload/medialibrary/8e7/2013_conley_newforeignpolfrontier_web_0-_1_.pdf).

62 The White House, National Strategy for the Arctic Region, 2014, disponible on line https://www.whitehouse.gov/sites/default/files/docs/nat_arctic_strategy.pdf.
} 
modifica en gran medida el escenario anterior. Esta nueva estrategia complementa, más no substituye, la directriz presidencial de $2009^{63}$ y está destinada a posicionar a Estados Unidos en la región para responder eficazmente a los nuevos desafíos y oportunidades derivados del aumento de la actividad en el Ártico. Como punto de partida, la estrategia de 2013 considera la región ártica como "peaceful, stable, and free of conflict" cuya gestión debe guiarse por el "spirit of trust, cooperation and collaboration, both internationally and domestically". A pesar de esta primera declaración de voluntad multilateralista, lo prioritario para Estados Unidos sigue siendo defender sus intereses en la región, lo que incluye "providing for the security of the United States; protecting the free flow of resources and commerce; protecting the environment; addressing the needs of indigenous communities; and enabling scientific research." No obstante, incorpora como nuevo objetivo el de convertir el Ártico en una región "where economic and energy resources are developed in a sustainable manner that also respects the fragile environment and the interests and cultures of indigenous peoples."

En este contexto se identifican tres líneas prioritarias de acción. Por un lado, en una perspectiva nacional, la estrategia va dirigida a reforzar la seguridad en la protección de los intereses nacionales. Por otro lado, las otras dos líneas de acción tienen una dimensión más internacional y abogan por llevar a cabo una administración responsable de la región ártica y desarrollar la cooperación multilateral, consensuando la protección de los intereses colectivos e intensificando la acción en el ámbito bilateral y en los organismos internacionales.

Estas tres líneas se detallan en el plan para la implementación de la estrategia ártica adoptado el 30 de enero de $2014^{64}$. En lo que se refiere a la protección de los intereses y la seguridad nacional, se establecen cuatro acciones prioritarias: 1) Reforzar las infraestructura marítima y aérea y las capacidades estratégicas y de comunicación en el Ártico; 2) promover la concienciación pública respecto a la región del Ártico; 3) preservar las libertades de los mares en la región ártica, reforzando la capacidad de las autoridades federales para realizar operaciones marítimas y 4) desarrollar políticas de promoción de las energías renovables y de gestión responsable de los recursos

\footnotetext{
${ }^{63}$ O'ROURKE, R., Changes in the Arctic: Background and Issues for Congress, 2016, p. 7, disponible online en https://www.hsdl.org/?view\&did=796197.

${ }^{64}$ The White House, Implementation Plan for the National Strategy for the Arctic región, 2014, disponible online 
energéticos. La implementación de la segunda de las líneas prioritarias, la administración responsable de la región ártica, se desarrolla mediante cuatro acciones especificas: 1) Proteger el medio ambiente ártico y sus recursos naturales mediante la conservación de los ecosistemas y la prevención de vertidos de con acciones de contención y respuesta; 2) promover una gestión integrada para equilibrar su desarrollo económico, la protección ambiental y los valores culturales de su población; 3) fomentar la investigación científica y la utilización de los conocimientos tradicionales mediante el desarrollo de nuevos modelos para la previsión y predicción de las condiciones en el hielo marino y sus ecosistemas, y la mejora de la sostenibilidad, el bienestar y la herencia cultural y lingüística de las comunidades árticas y 4) cartografiar el conjunto de la región ártica. Finalmente, para el reforzamiento de la cooperación internacional se establecen las siguientes acciones: 1) Apoyar acuerdos para una gestión compartida del Ártico, especialmente en materia de prevención, preparación y respuesta frente a la contaminación por hidrocarburos, mecanismos de búsqueda y rescate, pesca no regulada en alta mar, transporte de contaminantes, especies invasoras e investigación científica; 2) reforzar el Consejo Ártico; 3) promover la adhesión de Estados Unidos al Convenio sobre Derecho del Mar y 4) cooperar con otras partes interesadas en la gestión del Ártico, impulsando la adopción del Código Polar por parte de la Organización Marítima Internacional (OMI). Estas han sido, de hecho, las áreas prioritarias en las que se ha centrado la acción del Consejo Ártico durante la última presidencia estadounidense entre 2015-2017 ${ }^{65}$, que ha reflejado su voluntad de "to emphasize the Arctic Council as a forum for facilitating Arctic states' cooperation on myriad issues of mutual interest within its current mandate" ${ }^{\prime 66}$.

La estrategia nacional para el Ártico del Presidente Obama se reforzó significativamente con dos nuevos documentos estratégicos de la Coast Guard ${ }^{67} \mathrm{y}$ del Department of Defense (DoD) ${ }^{68}$ adoptados en mayo y noviembre de 2013, respectivamente. Sin

\footnotetext{
${ }^{65}$ Arctic Council, Proposed U.S. Arctic Council Chairmanship Program 2015-2017. February 2015. "One Arctic: Shared Opportunities, Challenges, and Responsibilities", Senior Arctic Officials meeting, Whitehorse, Canada, 4-5.3.2015, disponible online en https://oaarchive.arcticcouncil.org/handle/11374/1433.

${ }_{66}$ The White House, National Strategy for the Arctic Region, 2013. http://www.whitehouse.gov/sites/default/files/docs/nat_arctic_strategy.pdf.

${ }^{67}$ US Coast Guard, The US Coast Guard's Vision for operating in the Arctic Region, disponible online en https://www.uscg.mil/seniorleadership/docs/cg_arctic_strategy.pdf.

68 US Department of Defense, Arctic Strategy, 2013, disponible online en http://www.defense.gov/Portals/1/Documents/pubs/2013_Arctic_Strategy.pdf.
} 
embargo, hay una diferencia de tono entre el enfoque de la Coast Guard, que ve el Ártico como una región en profunda transformación, y el del DoD, mucho más tradicional ${ }^{69}$.

El documento estratégico de la Coast Guard tiene una visión más pro-futuro, centrada en el reconocimiento de unas circunstancias distintas y cambiantes en la región y la necesidad de planificar de manera anticipada su gestión. Así, la estrategia se abre con la afirmación sobre el carácter ártico de Estados Unidos y su predisposición para consensuar su gestión con sus socios en la región y se destacan los cambios generados por el cambio climático en términos de la apertura de nuevas rutas marítimas y la disponibilidad de nuevos recursos. Es importante destacar que el documento insiste sobremanera en la necesidad de cooperar a nivel internacional para mejorar la gobernanza del Ártico. Para la Coast Guard, esta acción estratégica debe dirigirse a asegurar las condiciones para el desarrollo de la actividad marítima y la gestión ambientalmente responsable en el Ártico. Para ello, prioriza la supervisión de las actividades relacionadas con la modernización y la mejora de la gobernabilidad del Ártico mediante la colaboración en las acciones colectivas y el reforzamiento de las asociaciones y partenariados existentes entre los sectores público y privado, incluyendo a las autoridades competentes a nivel nacional y las instancias de cooperación internacionales, como el Consejo Ártico, la OMI o el Consejo Circumpolar Inuit.

Por otro lado, la estrategia del DoD, más conservadora, está diseñada para salvaguardar los intereses de seguridad nacional. Para ello se establecen esencialmente dos grandes objetivos: Garantizar la seguridad y la cooperación en defensa, por un lado y prepararse para responder a una amplia gama de desafíos para mantener la estabilidad en la región, por el otro. Ello incluye desde la defensa antimisiles y los sistemas de alerta temprana o el despliegue de sistemas marítimos y aéreos estratégicos hasta las acciones de disuasión estratégica. Además de garantizar la seguridad en la región, el DoD tiene un mandato más técnico, que comprende la mejora de la cartografía náutica y la elaboración de indicadores del cambio climático en la región, aspectos especialmente relevantes para la industria del petróleo y el gas mar adentro. El DoD tiene también un papel clave en el ámbito de la seguridad marítima, esto es, con respecto de las operaciones de búsqueda y salvamento y en las acciones de respuesta a los vertidos de

\footnotetext{
${ }^{69}$ EBINGER, CH., BANKS, J.P., SCHACKMANN, A., Offshore Oil and Gas Governance in the Arctic.
} A Leadership Role for the U.S., ob.cit., p. 14. 
petróleo en aguas árticas. Esta perspectiva del DoD es la que ha reflejado tradicionalmente la US Navy Arctic Roadmappara el periodo 2010-2014 y 2014-2030, que planifica las actividades de este cuerpo en el Ártico, en el corto, medio y largo $\operatorname{plazo}^{70}$.

Otro punto de inflexión se da en enero de 2015, cuando el Presidente Obama adopta la Executive OrderEnhancing Coordination of National Efforts in the Arctic ${ }^{71}$. El punto de partida es claro: se trata de preservar el valor estratégico, ecológico, cultural y económico que tiene la región para el país. Dos factores impulsan la adopción de esta orden. En primer lugar, Estados Unidos se preparaba para asumir la presidencia del Consejo Ártico en abril de 2015 para el período 2015-2017. En segundo lugar, las diferencias políticas en torno al desarrollo económico de Alaska habían aumentado considerablemente y mientras que el gobierno de este estado exigía un modelo más dinámico vinculado con la explotación de los recursos de hidrocarburos, desde Washington se priorizaba una agenda climática más enfocada a la protección ambiental. Ambos elementos explican el viaje del presidente Obama a Alaska en 2015, viaje histórico en sí mismo porque era la primera vez que un presidente visitaba la región ${ }^{72}$.

Durante todos estos años, sin embargo, el desarrollo de estos objetivos se ha visto condicionado por la dispersión del entramado institucional necesario para su ejecución, auténtico talón de Aquiles de la política ártica estadounidense. La coordinación de estas acciones ha sido una tarea muy compleja, en la que han intervenido más de cuarenta departamentos y agencias federales, todos ellos con algún papel en la formulación y aplicación de la política ártica. De hecho, desde la creación de la US Arctic Research Commission (USARC) en $1984^{73}$ hasta el mismo nombramiento de un Representante

\footnotetext{
${ }^{70}$ US Navy, SER No. 9/9U103038, US Navy Arctic Roadmap 2010-2014, 10.11.2009, disponible online en http://greenfleet.dodlive.mil/files/2010/08/US-Navy-Arctic-Roadmap-11-10-09.pdf; US Navy, Updated Navy Arctic Roadmap for 2014-2030, 24.2.2014, disponible online en http://greenfleet.dodlive.mil/files/2014/02/USN-Arctic-Roadmap-2014.pdf.

${ }^{71}$ The White House, Executive Order 13689: Enhancing Coordination of National Efforts in the Arctic, 21.1.2015, disponible online en https://obamawhitehouse.archives.gov/the-pressoffice/2015/01/21/executive-order-enhancing-coordination-national-efforts-arctic

${ }^{72}$ US Arctic Executive Steering Committee, 2015 Year in Review. Progress Report on theimplementation of the National Strategy for the Arctic Region, 2016, pp. 4-15, disponible online en https://obamawhitehouse.archives.gov/sites/whitehouse.gov/files/documents/Progress\%20Report\%20on $\% 20$ the $\% 20$ Implementation $\% 20$ of $\% 20$ the $\% 20$ National $\% 20$ Strategy $\% 20$ for $\% 20$ the $\% 20$ Arctic $\% 20$ Region .pdf.

73 The White House, Executive Order 12501: Arctic research, 50 FR 4191, disponible online en https://www.archives.gov/federal-register/codification/executive-order/12501.html.
} 
Especial para el Ártico con rango de embajador en $2014^{74}$, la política estadounidense en torno al Ártico se ha resentido de esta falta de coordinación interna ${ }^{75}$.Como han indicado algunos comentaristas, "adding more layers and levels of governmental coordination simply wasn't advancing U.S. Arctic policy; instead, the current process created more meetings with a growing number of attendees without actionable outcomes" ${ }^{76}$.Tal superposición y redundancia refleja las dificultades prácticas de la política ártica estadounidense excesivamente fragmentada y de su encaje en el proceso interinstitucional ${ }^{77}$.

Este periodo de relativa implicación en la región puede finalizar abruptamente con la llegada de Donald Trump a la presidencia norteamericana. Aunque la administración del presidente Trump aun no ha hecho pública ninguna declaración específica con respecto a la futura estrategia de Estados Unidos para el Ártico, parece probable un retroceso a su política anterior de ignorar la región, excepto en lo que afecta a los recursos de hidrocarburos en Alaska.

Por un lado, a nivel internacional, la retirada de Estados Unidos del Acuerdo de Paris, notificada formalmente el pasado 4 de agosto de 2017 al Secretario General de Naciones Unidas ${ }^{78}$ no permite grandes dosis de optimismo, ya que seguramente se traducirá en una falta de apoyo a las medidas de mitigación y adaptación al cambio climático que son indispensables para la conservación de los ecosistemas y las comunidades indígenas en el Ártico. Además esto incidirá en los trabajos del Consejo Ártico y de otros foros multilaterales, en los que seguramente la acción de la

\footnotetext{
74 Arctic Law \& Policy Institute, “Arctic Law and Policy Year in Review: 2014”, Washington Journal of Environmental Law and Policy, Vol. 5 (1), 2015, pp. 100-174, p. 101.

75 US Government Accountability Office, Report to Congressional Request, Arctic Issues: Better Direction and Management Voluntary Recommendation Could Enhance US Arctic Council Participation, 2014, GAO-14-435, pp. 22 y ss.A diferencia de las directrices de 1971 y 1994, la Directriz de 2009 se remitió a las 24 agencias estatales existentes en aquel momento, poniendo en evidencia la dificultad de la gestión interdepartamental y diluyendo los mecanismos de coordinación. La Executive Order 13689: Enhancing Coordination of National Efforts in the Arctic de 21 de enero 2015 que crea el Arctic Executive Streering Committee (AESC) constituye un último intento fallido de racionalizar esta situación.

${ }^{76}$ CONLEY, H., The New Foreign Policy Frontier: US interests and actors in the Arctic, ob.cit., p. 21.

${ }^{77}$ EBINGER, CH., BANKS, J.P., SCHACKMANN, A., Offshore Oil and Gas Governance in the Arctic. A Leadership Role for the U.S., ob.cit., pp. 34 y ss.; HULTS, D., "Environmental Regulation at the Frontier: Government Oversight of Offshore Oil Drilling North of Alaska", ob.cit., pp. 823-826; ARRUDA, G.M., "Arctic governance regime: the last frontier for hydrocarbons exploitation", International Journal of Law and Management, Vol. 57 (5), 2015, pp. 498-521.

${ }^{78}$ El texto de la carta remitida por el presidente Trump al Secretario General de Naciones Unidas puede verse en MATHIESEN, K., "Trump letter to UN on leaving Paris climate accord - in full", ClimateHome, 8.8.2017, disponible online en http://www.climatechangenews.com/2017/08/07/trump-tells-un-intentionleave-paris-climate-accord-full/.
} 
administración estadounidense se orientará de nuevo hacia el unilateralismo. Por otro lado, a nivel interno, como se comenta más adelante, el presidente Trump ya anunciado su pleno soporte al desarrollo del potencial energético convencional de Estados Unidos, con una especial referencia a los hidrocarburos del Ártico, tal y como demuestran sus primeras decisiones a este respecto. Parece pues que por el momento, la posición de la administración Trump puede resumirse en una de las frases que éste pronunció con ocasión de la adopción de sus primeras medidas el pasado 28 de abril de 201779: "So we can't spend too much time talking about drilling in the Arctic, right? But we're opening it $u p, " 80$.

\section{El conflicto entre el gobierno federal y el estado de Alaska en torno a la exploración y explotación mar adentro de petróleo ártico}

El primer aspecto que interesa destacar es el conflicto que la prospección y explotación mar adentro de los hidrocarburos del Ártico ha generado entre la administración federal y el estado de Alaska ${ }^{81}$. Se trata de determinar cuál es el nivel de gobierno que garantiza una mayor protección del medio ambiente ártico y de los recursos naturales en alta mar y en la plataforma continental, a la vez que permite un desarrollo económico sostenible de esta región.

De modo general, es el Congreso de Estados Unidos quien tiene la competencia legislativa sobre las aguas y el lecho marino del Océano Ártico, que ha ejercido mediante la adopción de diversas leyes que afectan a la protección del medio ambiente, el transporte o la pesca ${ }^{82}$. El ejecutivo y las agencias reguladoras federales son

\footnotetext{
79 The White House,Presidential Executive Order Implementing an America-First Offshore Energy Strategy, 28.4.2017, disponible enhttps://www.whitehouse.gov/the-press-office/2017/04/28/presidentialexecutive-order-implementing-america-first-offshore-energy

${ }^{80}$ The White House, Office of the Press Secretary, Remarks by President Trump at Signing of Executive Order on an America-First Offshore Energy Strategy, 28.4.2017, disponible online en https://www.whitehouse.gov/the-press-office/2017/04/28/remarks-president-trump-signing-executiveorder-america-first-offshore.

${ }^{81}$ LINXWILER, J.D., "Federal Oil and Gas Leasing in Alaska", en Rocky Mountain Mineral Law Foundation (Ed.), Law on Federal Oil and Gas Leases, LexisNexis, 1985 (puesta al dia anual). Este capitulo está disponible online en https://www.google.es/search?q=Alaska+National+Interest+Lands+Conservation+Act+AND+arctic+oil+

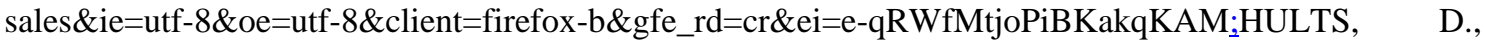
"Environmental Regulation at the Frontier: Government Oversight of Offshore Oil Drilling North of Alaska", ob.cit., pp. 769-780.

${ }^{82}$ Coastal Zone Management Act, 16 U.S.C. 1451, Marine Protection, Research, and Sanctuaries Act, 16 U.S.C. 1431, Marine Mammal Protection Act, 16 U.S.C. 1361, Fishery Conservation and Management
} 
responsables de la promulgación de los reglamentos de ejecución en conformidad con estas leyes. Sin embargo, en virtud de la Submerged Lands Act (SLA) de $1953^{83}$, el gobierno federal comparte con los estados la jurisdicción sobre su mar territorial, así como la responsabilidad de la gestión de sus recursos ${ }^{84}$.La ley reconoce la competencia de los estados sobre el lecho marino, las aguas y los recursos naturales que se encuentran dentro de las tres millas náuticas desde la línea de base y con ello, su poder expreso para arrendar, desarrollar y administrar los recursos en este espacio ${ }^{85}$. El gobierno federal conserva la competencia para la regulación de las actividades de explotación de los recursos marinos que se encuentran más allá de las tres millas náuticas, en el lecho marino o la columna de agua y en especial, los de la plataforma continental, que son administrados por el Department of Interior (DoI) y en su nombre por el Bureau of Ocean Energy Management (BOEM).

La Outer Continental Shelf Land Act (OCSLA), del mismo año ${ }^{86}$, también confiere al gobierno federal el control de las actividades que se llevan a cabo en la plataforma continental, que sujeta a ciertas salvaguardas ambientales y a las necesidades nacionales. Para ello extiende la aplicación de la legislación federal a ciertas infraestructuras en territorio estatal, donde permite la aplicación de la legislación estatal si no es incompatible con la federal ${ }^{87}$. Conforme a esta ley, corresponde al gobierno federal la concesión de derechos mineros y de extracción de hidrocarburos en la plataforma continental y, por tanto, el desarrollo de los programas quinquenales de arrendamientos para la explotación de hidrocarburos, que son ejecutados por el BOEM. En particular, la OCSLA establece cuatro fases diferenciadas para el desarrollo de la explotación del petróleo y el gas mar adentro, que requieren, cada una de ellas, una autorización expresa: la fase de planificación mediante un programa quinquenal de arrendamiento de áreas de prospección y explotación en la plataforma continental; la

Act, 16 U.S.C. 1801, Endangered Species Act, 16 U.S.C. 1531, and National Environmental Policy Act, 42 U.S.C. 4331.

8343 U.S.C. 1301-1303.

${ }^{84}$ CANUEL, E., "Alaska and offshore hydrocarbon extraction. A legal and socio-economic review", en PELAUDEIX, C.-BASSE, E.M., (Ed.), Governance of Arctic Offshore Oil and Gas, Routledge, 2017, pp. 95-103.

${ }^{85}$ Ibid. 1301.

8643 U.S.C. 1311-1356.

${ }^{87} 43$ U.S.C. 1331,1332 y 1333. 
fase de comercialización de estos arrendamientos; la fase de exploración y prospección de las áreas arrendadas; y finalmente, la fase de desarrollo y explotación de la actividad. Asimismo, la National Environmental Policy Act (NEPA) ${ }^{88}$ concede un papel muy significativo al gobierno federal, ya que es el DoI quien compila las declaraciones de impacto ambiental que requiere cada una de estas fases, de acuerdo con las que se determinan las áreas susceptibles de ser explotadas y que resultan aptas para el otorgamiento de las concesiones, que se agrupan posteriormente en los programa de arrendamiento quinquenales.

Por otro lado, la Coastal Zone Management Act (CZMA) ${ }^{89}$ de 1972 persigue la protección y desarrollo de las zonas costeras bajo competencia estatal mediante la adopción de programas de gestión de las zonas costeras adoptados por las autoridades estatales ${ }^{90}$. Como contrapartida al predominio del gobierno federal respecto a la explotación de la plataforma continental, cuando estas actividades se realizan dentro de las tres millas náuticas la ley exige que las actividades federales se lleven a cabo de manera coherente con estos programas estatales, lo que en principio ha de permitir compatibilizar esta explotación con la preservación de sus recursos naturales.

Así pues, a nivel federal, la SLA y la OCSLA establecen desde los años ochenta una suerte de "federalismo dual geográfico" 91 . Dentro de las tres millas náuticas, el papel de los estados es predominante, aunque no exclusivo. Éstos son responsables de las decisiones relativas a la gestión de los recursos, pero el gobierno federal conserva sus competencias sobre este espacio, que tradicionalmente se limitaban al control de inmigración, el control del cumplimiento de las leyes aduaneras, la navegación y funciones similares. Por el contrario, más allá de las tres millas náuticas, la responsabilidad principal recae en el gobierno federal, especialmente en lo que se

\footnotetext{
8842 U.S.C. 4331.

${ }^{89} 16$ U.S.C. 1451.

${ }^{90}$ Sin embargo, Alaska no dispone aún de un Programa de Gestión de las Zonas Costeras conforme a la CZMA, desde que en 2011 se rechazara la renovación del programa de 1977. Vid., National Oceanic and Athmospheric Administration, Alaska Coastal Management Program Withdrawal From the National Coastal Management Program Under the Coastal Zone Management Act (CZMA), Office of Federal Register, disponible online en https://www.federalregister.gov/documents/2011/07/07/201116987/alaska-coastal-management-program-withdrawal-from-the-national-coastal-management-programunder-the

${ }^{91}$ MILLERT, D.S., "Offshore Federalism: Evolving Federal-State Relations in Offshore Oil and Gas Development", Ecology Law Quartlerly, Vol. 11, 1984, pp. 401-450; RAZO, G.R, "The Seaweed Rebellion Revisited: Continuing Federal-State Conflict in OCS Oil and Gas Leasing", Willamette Law Review, Vol. 20, 1984, pp. 83-140.
} 
refiere a la gestión de hidrocarburos, si bien también es de aplicación parte de la legislación estatal de gestión de costas.

Sin embargo, debido a la situación de déficit presupuestario y a la excesiva dependencia de la industria petrolera que tradicionalmente ha mostrado el gobierno federal ${ }^{92}$, este sistema no parece haber conseguido preservar adecuadamente el interés que los estados han manifestado con respecto a la protección de su medio ambiente y sus recursos naturales frente a los efectos adversos de las actividades de prospección y explotación del petróleo. La tensión entre las diversas jurisdicciones evidencia así la dificultad de compaginar el logro de objetivos distintos a cargo del gobierno federal, como son el desarrollo de la industria del gas y del petróleo como motor de crecimiento económico y la protección de los recursos naturales dentro de su jurisdicción.

Los conflictos entre los estados y el gobierno federal han sido recurrentes en la historia reciente de la explotación mar adentro de petróleo y gas en Estados Unidos. Los tribunales nacionales han mantenido de manera reiterada que no cabe reconocer derechos distintos a los federales en la plataforma continental más allá de las tres millas náuticas ${ }^{93}$. Por su parte, la Corte Suprema se ha pronunciado en diversas ocasiones en las que los estados han impugnado el ejercicio de estas competencias por parte del gobierno federal en su frontera marítima ${ }^{94}$. Su posición ha sido tradicionalmente la de considerar que la acción del gobierno federal debe ser "consistente en la medida de lo posible" con las exigencias de la CZMA, pero ha querido evitar una interferencia excesiva por parte de los estados en la gestión de los recursos más allá de las tres millas náuticas. Así, este alto tribunal, que en 1947 ya había establecido la competencia del gobierno federal sobre todas las áreas sumergidas que se extienden desde la costa hacia

\footnotetext{
${ }^{92}$ HULTS, D., "Environmental Regulation at the Frontier: Government Oversight of Offshore Oil Drilling North of Alaska", ob.cit., pp. 790-794; KALEN, S., "Cruise Control and Speed Bumps: Energy Policy and Limits for Outer Continental Shelf Leasing", Environmental \&Energy Law\& Policy Journal, Vol. 7, 2012, pp. 156-189, pp. 161-167.

${ }^{93}$ US Court of Appeals, Ninth Circuit, Native Village of Eyak et al. v. Trawler Diane Marie, Inc., $\mathrm{n}^{\circ} .97-$ 35944, (527 US 1003), 9.9.1998.

${ }^{94}$ US Supreme Court, United States v. Louisiana, 363 U.S. 1 (1960); United States v. Florida, 363 U.S. 121 (1960); United States v. Maine, 475 U.S. 89 (1986); United States v.Maine, 469 U.S. 504 (1985); United States v. California, 381 U.S. 139 (1965); United States v. Alaska, 521 U.S. 1 (1997). Para un comentario detallado, Vid., REED, M.W., Shore and Sea Boundaries, Vol. 3, U.S. Department of Commerce, National Oceanic and Atmospheric Administration, Coastal Services Center, Office of Coast Survey - U.S. Government Printing Office, Washington, 2000.
} 
la plataforma continental ${ }^{95}$, confirmó en 1960 la regla de las tres millas náuticas ${ }^{96}$. En 1997, a raíz de una controversia entre el gobierno federal y Alaska relativa al ejercicio de las competencias sobre los espacios adyacentes a la costa ártica, en la que el primero reclamaba el derecho de arrendar el territorio en el Mar de Beaufort para la explotación de minerales e hidrocarburos, la Corte Suprema se pronunció de nuevo en el sentido de que, conforme a la SLA, corresponde al estado de Alaska únicamente la gestión del espacio comprendido dentro de las tres millas desde la línea de base, mientras que el territorio adyacente más allá de esta distancia es de jurisdicción federal ${ }^{97}$.

Por su parte, los órganos legislativos y el ejecutivo del estado de Alaska tienen poca autoridad sobre los territorios federales y, especialmente cuando se trata de actividades económicas o de conservación del medio ambiente, su poder se solapa significativamente con la legislación federal del Estado ${ }^{98}$. Las diferencias entre el gobierno federal y el ejecutivo estatal de Alaska ${ }^{99}$ han sido especialmente relevantes respecto al alcance de la Alaska National Interest Lands Conservation Act (ANILCA) de $1980^{100}$, por la que se designan las tierras federales de Alaska como unidades de sistema de conservación, lo que incluye los parques federales, refugios y zonas de especial protección en Alaska para los que se establece un régimen especial de gestión (National Wilderness Preservation System).

Uno de los objetivos principales de la ANILCA era completar la asignación de tierras federales en el estado de Alaska, proceso iniciado con la Alaska Statehood Act de 1958 (ASA) ${ }^{101}$ y la Alaska Native Claims Settlement Act (ANCSA) de $1971^{102}$, un proceso que el estado nunca llegó a culminar ${ }^{103}$. A los efectos que interesan, además de los

\footnotetext{
${ }^{95}$ US Supreme Court, United States v. California, 332 U.S. 19, 36 (1947). Ver también United States v. Louisiana, 339 U.S. 699 (1950) y United States v. Texas, 339 U.S. 707 (1950). Ver por todos, REED, M.W., Shore and Sea Boundaries, ob.cit.

96 US Supreme Court, United States v. Louisiana, 363 U.S. 1 (1960), 31.5.1960.

${ }^{97}$ US Supreme Court, United States v. Alaska, 521 U.S. 1 (1997), 19.6.1997.

${ }^{98}$ Para un estudio detallado, Vid., LINXWILER, J.D., "Federal Oil and Gas Leasing in Alaska”, ob.cit.

${ }^{99}$ El estado de Alaska tiene poca legislación específica sobre el Ártico, que principalmente consiste en la Alaska Coastal Management Act (ACMA) de 1977 (16 U.S.C. 1451-1464,) y la Alaska National Interest Lands Conservation Act (ANILCA) de 1980 (16 U.S.C. 3101-3233 y 43 U.S.C. 1635), algo que seguramente cambiará a medida que la actividad económica aumente a lo largo de su costa norte.

10016 U.S.C. 3101-3233.

10148 U.S.C. note prec. $\S 21$.

10243 U.S.C. 1601-1628.

${ }^{103}$ LINXWILER, J.D., "Federal Oil and Gas Leasing in Alaska", ob.cit., p. 4 y ss.
} 
campos petrolíferos ubicados en los territorios estatales de la Bahia de Prudhoe (las denominadas North Slope Lands), la ley autorizaba los arrendamientos para la explotación de petróleo y gas en los territorios bajo jurisdicción federal (las denominadas Non-North Slope Lands): en el oeste de la National Petroleum Reserve in Alaska (NPRA) al norte de los $68^{\circ}$ latitud norte y al este, en el ArcticNational Wildlife Refuge System (ANWRS). Para ello se requería que se determinara que esta actividad no era incompatible con el objetivo de protección para el que se creó y mediara la autorización expresa del Congreso. La aplicación de la ley a lo largo de estos años ha generado una serie de tensiones entre el gobierno federal y estatal respecto al ejercicio de las competencias para autorizar la prospección y la explotación petrolera y gasística en este estado.

Esta situación se refleja claramente en el asunto Amoco Production Co. v. Village of Gambell $^{104}$. Este caso se inició con una demanda planteada por dos comunidades nativas ante el Tribunal de Distrito de Alaska ${ }^{105}$ por el incumplimiento de la ANILCA, que exige la evaluación del impacto ambiental antes de autorizar ciertos usos de los espacios incluidos en su ámbito de aplicación y la presentación de alternativas para eliminar tales efectos, además de requerir una serie de salvaguardas procesales para garantizar los derechos de participación a las partes interesadas. El Tribunal de Distrito consideró que la ANILCA era la norma aplicable al caso y que, como alegaban los demandantes, la administración federal la había vulnerado en el momento en que autorizó los arrendamientos para la exploración de petróleo a empresas privadas. Sin embargo, denegó la adopción de medidas cautelares por entender que la exploración no causaba un daño significativo en los intereses que la ley protegía (en este caso, los usos o recursos de subsistencia de las poblaciones indígenas y las comunidades rurales) y que existía un fuerte apoyo público para este proyecto. Posteriormente, el Tribunal de Apelaciones para el Noveno Circuito, si bien confirmó la aplicación de la ANILCA, revirtió parcialmente este fallo afirmando la exigencia de medidas cautelares, puesto que consideró que podía presumirse la posibilidad del daño cuando la agencia responsable no evaluaba minuciosamente el impacto ambiental de la actuación que se proponía llevar a cabo ${ }^{106}$.

\footnotetext{
${ }^{104}$ US Supreme Court, Amoco Production v. Village of Gambell, 480 U.S. 531 (1987), 24.3.1987. ${ }^{105}$ Alaska District Court, People of Gambell v. Clark, 746 F.2d 572 (1984) (Gambell I) 2.11.1984.

${ }^{106}$ US Court of Appeals, Ninth Circuit, People of Gambell v. Hodel, 774 F.2d 1414 (1985) (Gambell II), 25.10.1985.
} 
En su pronunciamiento de 1987, en cambio, la Corte Suprema determinó que las disputas petroleras sobre la plataforma continental de Alaska no estaban regidas por la ANILCA, que sólo permite regular hasta tres millas náuticas desde la línea de base. Así, la Corte indicó claramente que la ley "applies only to federal lands within the State of Alaska's boundaries, since the Act defines "public lands" to mean federal lands situated "in Alaska", which phrase has a precise geographic/political meaning that can be delineated with exactitude to include coastal waters to a point three miles from the coastline, where the OCS [Outside Continental Shelf] commences." Añadía que "the Act contains the lone express reference to the OCS which is intended to establish that section's inapplicability to the $O C S^{\prime 107}$. Como resultado, este caso permitió que las compañías petroleras comenzaran las actividades exploratorias a pesar de la oposición de la población nativa de Alaska y de las organizaciones ambientales.

La Corte Suprema ha mantenido tradicionalmente esta posición a lo largo de los años, y en una reciente sentencia de 22 de marzo $2016^{108}$ rechazó de nuevo la interpretación del Tribunal de Apelaciones para el Noveno Circuito respecto del alcance de la competencia federal, por estimar que la legislación federal aplicable a Alaska reconoce un tratamiento específico de este estado que se justifica por su particularidad y que lo diferencia del que se aplica al resto del país.

En este asunto se cuestionaba la sentencia del Tribunal de Apelaciones para el Noveno Circuito de 14 de octubre de 2014, que declaraba de nuevo la jurisdicción de la ANILCA sobre el territorio cubierto por el Servicio Forestal federal ${ }^{109}$. Su decisión, en relación a un recurso contra una sentencia del Tribunal de Distrito de Alaska ${ }^{110}$, consideraba que los territorios estatales, de los pueblos nativos o que eran propiedad privada incluidos dentro de las unidades de sistema de conservación de la ANILCA, no estaban sujetos a la normativa federal, aplicable únicamente a los territorios federales dentro de estas unidades. La norma federal (en el caso concreto se trataba de la prohibición de utilizar aerodeslizadores en aguas estatales) solo era de aplicación a las

\footnotetext{
${ }^{107}$ US Supreme Court, Amoco Production v. Village of Gambell, ob.cit., pp. 546-548.

${ }^{108}$ US Supreme Court, Sturgeon v. Frost, 577 U.S. (2016), 22.3.2016.

${ }^{109}$ U.S. Court of Appeals, Ninth Circuit, John Sturgeon v. Masica et al., 768 F.3d 1066, 1069-70 (2014), 6.10.2014.

${ }^{110}$ Alaska District Court, Sturgeon v. Masica, no 3:11-cv-0183 (2013), 30.10.2013.
} 
tierras de propiedad federal y a las aguas administradas por el Servicio de Parques federal, así como a todas las aguas navegables en los parques nacionales.

La Corte Suprema revocó la decisión del Tribunal de Apelaciones para el Noveno Circuito por no ser conforme con la literalidad y el contexto de la ANILCA. Así, entiendió que "those Alaska-specific provisions reflect the simple that Alaska is often the exception, not the rule. Yet the reading below would prevent the Park Service from recognizing Alaska's unique conditions. Under that reading, the Park Service could regulate "non-public" lands in Alaska only through rules applicable outside Alaska as well. (...) Looking at ANILCA both as a whole and with respect to Section 103(c), the Act contemplates the possibility that all the land within the boundaries of conservation system units in Alaska may be treated differently from federally preservation areas across the country, and that "non-public" lands within the boundaries of those units may be treated differently from "public" lands within the unit. Under the Ninth Circuit's reading of Section 103(c), however, the former is not an option, and the latter would require contorted and counterintuitive measures" 111 .

Los conflictos existen también en el sentido contrario. En el caso de Alaska, un estado de tradición marcadamente republicana, el gobierno estatal y la industria petrolera han abogado históricamente por la realización de actividades exploratorias y de prospección de petróleo y gas en la plataforma continental adyacente al Arctic National Wildlife Refuge System, tal y como se han venido haciendo desde hace años en la parte central del North Slope. Incluso algunas de las comunidades indígenas, con una participación importante en la propiedad de estos territorios a través de la Kaktovik Inupiat Corp. y la Arctic Slope Regional Corp., han apoyado este tipo de actividades, si bien, conscientes de los riesgos que conlleva la explotación mar adentro, limitadas hasta ahora a la explotación tierra adentro.

Es relevante en este punto mencionar la demanda ante el Tribunal de Distrito de Alaska que interpuso el gobierno de Alaska contra el DoI ${ }^{112}$ por la suspensión de los planes de prospecciones sísmicas para la localización de bolsas de petróleo y gas en la plataforma continental adyacente al Arctic National Wildlife Refuge System. En particular, Alaska contestaba la denegación de su programa de pruebas sísmicas por considerar que esta área seguía abierta a la actividad exploratoria, independientemente de cualquier

\footnotetext{
${ }^{111}$ Ibid., pp. 14-15.

${ }^{112}$ Alaska District Court, State of Alaska v. Sally Jewell et al., n 3:14-cv-00048, 22.7.2016.
} 
producción real de petróleo o gas natural. De acuerdo con las autoridades de Alaska, la ANILCA exigía equilibrar el interés nacional en la protección de los recursos naturales de Alaska con las necesidades económicas y de infraestructura del estado, y muy especialmente con la exploración y explotación de sus vastos recursos minerales y energéticos. Consideraba, de una parte, que la ANILCA permitía estas actividades exploratorias y requería de la autoridad federal la aprobación de las propuestas de terceros, en este caso el estado de Alaska, para desarrollarlas. De la otra, estimaba que ésta era una actividad permitida conforme al Arctic National Wildlife Refuge Coastal Plain Resource Assessment ${ }^{113}$, que consiente la actividad exploratoria cuando no cause efectos adversos significativos sobre los peces y la fauna silvestre y otros recursos. Sin embargo, en una decisión muy aplaudida por los grupos ambientalistas, el Tribunal de Distrito se pronunció el 21 de julio de 2015 en contra de esta petición y confirmó la interpretación de las autoridades federales, que entienden que su autoridad para aprobar este tipo de exploraciones en este territorio conforme a la ANILCA expiró en 1987, fecha en que los estados debían presentar sus evaluaciones definitivas conforme a la ley, y que, en ausencia de tal evaluación -éste era el caso de Alaska-, su interpretación no permite la extensión en el tiempo de este mandato. Consideró además que la ANILCA permite la limitación de la actividad exploratoria como uno de los mecanismos posibles para lograr el objetivo de reducir los impactos adversos de la producción de petróleo y gas en esta área.

Treinta años después, por tanto, el fondo de la cuestión sigue siendo el alcance y el equilibrio de la competencia del gobierno federal y estatal para administrar los programas para la explotación de los hidrocarburos en la plataforma continental. Los distintos conflictos planteados ante el Tribunal de Apelaciones para el Noveno Circuito y ante la Corte Suprema, frecuentemente con decisiones divergentes, afectan el desarrollo actual y futuro de estos recursos y reflejan en cierto modo la ineficacia actual de los instrumentos jurídicos de que disponen los estados. En el centro de estas disputas se encuentran intereses nacionales que cobran ahora, bajo la presidencia de Donald Trump, una importancia inusitada. Por un lado, el gobierno federal y la industria petrolera tratan de intensificar el desarrollo de la explotación mar adentro de hidrocarburos con el fin de promover la independencia energética y aumentar los

\footnotetext{
11316 U.S.C. 3142 .
} 
ingresos y beneficios de los programas quinquenales de arrendamiento de la plataforma continental. Por otro lado, los estados, generalmente junto con los grupos ambientalistas y las comunidades autóctonas afectadas por la explotación de estos recursos, buscan preservar las zonas costeras amenazadas, a la vez que reclaman más autonomía para decidir sobre el modelo de desarrollo económico del estado ${ }^{114}$.

\section{El lento e inacabado proceso de limitación de los arrendamientos para la exploración y explotación mar adentro en el Ártico}

Como se ha señalado, la Sección 18 de la OCSLA requiere de la administración federal la elaboración de un programa de arrendamiento quinquenal para la ordenación de los arrendamientos en alta mar, en el que se establecen el calendario, tamaño y localización de dichos arrendamientos ${ }^{115}$. Estos programas, nueve desde 1980, se elaboran teniendo en cuenta las necesidades energéticas nacionales durante el período que abarcan y el alcance de los potenciales impactos económicos, sociales y ambientales asociados al desarrollo de estas explotaciones.

Sin embargo, hasta bien entrada la década del 2000 no hubo en Estados Unidos una planificación federal real de las explotaciones petrolíferas y gasísticas en el Ártico ${ }^{116}$. En 2008, el Presidente G. W. Bush y el Congreso eliminaron las moratorias existentes sobre los arrendamientos en diversas zonas de la plataforma continental ${ }^{117} \mathrm{y}$, como consecuencia, el programa federal de arrendamiento de la plataforma continental para el periodo 2007-2012 amplió sustancialmente las concesiones en los mares de Chukchi y Beaufort $^{118}$.

\footnotetext{
${ }^{114}$ KALEN, S., "Cruise Control and Speed Bumps: Energy Policy and Limits for Outer Continental Shelf Leasing”, ob.cit., pp. 167-178.

115 OSCLA, Sección 1344(a), (e).

${ }^{116}$ Department of the Interior and related Agencies Appropriatioin Act, 2004, disponible online enhttps://www.congress.gov/bill/108th-congress/house-bill/2691

117 "Memorandum on Modification of the Withdrawal of Certain Areas of the United States Outer Continental Shelf from Leasing Disposition”, 44 Weekly Comp. Pres. Doc. Vol. 44, 14.7.2008, p. 986.

${ }^{118}$ US Department of Interior Minerals Management Service, Proposed Final Program Outer Continental Shelf Oil and Gas Leasing Program 2007-2012, 2007, disponible online en https://www.boem.gov/Oiland-Gas-Energy-Program/Leasing/Five-Year-Program/MMSProposedFinalProgram2007-2012-pdf.aspx
} 
La llegada de la administración Obama trajo consigo la revisión del programa y una pequeña reducción inicial de las zonas de arrendamiento ${ }^{119}$. En pleno debate sobre las consecuencias del accidente del Deepwater Horizon esta programación había generado una importante oposición por parte de diversos grupos de interés, que cuestionaron la calidad y al alcance de las evaluaciones de impacto ambiental realizadas por la administración federal. En 2010, como respuesta a estas críticas, el presidente Obama restableció la moratoria en el Mar de Bering, que excluía las áreas ubicadas en la Bahía de Bristol de las operaciones de arrendamiento para la exploración y explotación de gas y petróleo hasta después de $2017^{120}$. Esta línea tuvo continuidad en el período 20122017, previéndose la apertura de sólo tres procedimientos de arrendamiento para la explotación en Alaska (en el Mar de Chukchi y la Ensenada de Cook en 2016 y en el Mar de Beaufort en 2017) ${ }^{121}$.

Más allá de estas primeras medidas, el proceso de suspensión de los arrendamientos en el Ártico se inicia propiamente en 2014 a raíz de la sentencia del Tribunal de Apelaciones para el Noveno Circuito de 22 de enero de $2014^{122}$, que había declarado no conformes a derecho los arrendamientos realizados en el Mar de Chukchi. El Tribunal consideró en esta ocasión que la declaración de impacto ambiental del DoI/BOEM solo había contemplado el mejor escenario posible en relación al daño ambiental, subestimando gravemente los riesgos de un vertido de petróleo y otros efectos conexos, lo que obligó a revisar los estudios del impacto ambiental de las perforaciones autorizadas y solicitadas.

En diciembre de este mismo año el Presidente Obama modificó la moratoria en el Mar de Bering para convertirla en indefinida ${ }^{123}$. Al cabo de un mes, en enero de 2015 se

${ }^{119}$ US Bureau of Ocean Energy Management, Regulation, and Enforcement, Revised Program, Outer Continental Shelf Oil and Gas Leasing Program, 2007-2012, 2010, disponible online en http://www.boem.gov/Oil-and-Gas-Energy-Program/Leasing/Five-Year-Program/RP-pdf.aspx.

120 The White House,Presidential Memorandum, "Withdrawal of Certain Areas of the United States Outer Continental Shelf from Leasing Disposition," 31.3.2010, disponible online en http://www.doi.gov/whatwedo/energy/ocs/upload/2010alaska-mem-rel.pdf.

${ }^{121}$ US Bureau of Ocean Energy Management, Proposed Final Outer Continental Shelf Oil \& Gas Leasing Program, 2012- 2017, 2012, disponible online en at http://www.boem.gov/uploadedFiles/BOEM/Oil_and_Gas_Energy_Program/Leasing/Five_Year_Progra m/2012-2017_Five_Year_Program/PFP\%2012-17.pdf.

${ }^{122}$ U.S. Court of Appeals, Ninth Circuit, Native Village of Point Hope v. Jewel, $\mathrm{n}^{\mathrm{o}}$ 1:08-cv-00004-

RRB, 22.1.2014, pp. 31 y 32.

123 The White House,Presidential Memorandum, Withdrawal of Certain Areas of the United States Outer Continental Shelf from Leasing Disposition, 16.12.2014, disponible online en 
produjo una nueva suspensión de los arrendamientos para 2016 y 2017 en el Mar de Chukchi y el Mar de Beaufort ${ }^{124}$. Aunque ésta decisión se justificaba supuestamente en unas difíciles condiciones del mercado y el bajo interés de la industria petrolera en la región, el Presidente citaba también, de manera expresa, la importancia crítica de estas áreas para la subsistencia de los pueblos indígenas de Alaska, así como para la protección de los mamíferos marinos y otras especies silvestres ${ }^{125}$.

En 2016 el gobierno federal adoptó el aún vigente programa de arrendamiento para 2017-2022, que incluye tres procedimientos de arrendamiento muy limitado en el Mar de Beaufort (2020), la Ensenada de Cook (2021), y en el Mar de Chukchi (2022) ${ }^{126}$. Sin embargo, resultado de la oposición de los sectores y de los grupos ambientalistas que consideraban excesivamente elevado el riesgo de un accidente en la zona y del proceso de revisión de los incidentes acaecidos desde 2012 en la plataforma de Shell en el Mar de Chukchi ${ }^{127}$, la administración Obama, en una de sus últimas acciones, adoptó dos

http://www.whitehouse.gov/the-press-office/2014/12/16/presidential-memorandum-withdrawal-certainareas-united-states-outer-con.

${ }^{124}$ The White House,Presidential Memorandum, Withdrawal of Certain Areas of the United States Outer Continental Shelf Offshore Alaska from Leasing Disposition, 27.1.2015, disponible online en http://www.whitehouse.gov/the-press-office/2015/01/27/presidential-memorandum-withdrawal-certainareas-united-states-outer-con.

${ }^{125}$ US Department of the Interior, Interior Department Cancels Arctic Offshore Lease Sales, Press Release, 16.10.2015, disponible online en https://www.doi.gov/pressreleases/interior-department-cancelsarctic-offshore-lease-sales.

${ }^{126}$ US Bureau of Ocean Energy Management, 2017-2022 Outer Continental Shelf Oil and Gas Leasing Proposed Program, 2016, disponible en https://www.boem.gov/2017-2022-OCS-Oil-and-Gas-Leasing$\mathrm{PFP} /$.

${ }^{127}$ Desde 2009, cuando presentó su primer plan de exploración, hasta que abandonó las prospecciones en el Mar de Chukchi en 2015, la actividad de Shell se ha visto rodeada de la polémica. En 2010 el Tribunal Federal de Distrito en Alaska determinó que los arrendamiento concedidos a Shell en 2007 y 2008 vulneraban la legislación ambiental estadounidense, al no haberse considerado las particularidades de la zona en cuestión. En 2011, Shell presentó un nuevo plan de perforación para el Mar de Chukchi y el Mar de Beaufort. Después de diversos desacuerdos con la EPA, obtuvo los permisos en 2012, pero limitados al Mar de Chukchi. Este mismo año, antes ya de empezar su actividad, el buque de perforación de Shell, el Noble Discoverer sufrió un accidente y quedó varado y sin control en Dutch Harbor (Alaska). Más adelante, la Guardia Costera lo detuvo en Seward por los problemas que presentaba en los sistemas de descarga de contaminantes. Entretanto, otra plataforma de Shell que está perforando cerca de la isla de Kodiak quedó también a la deriva durante una tormenta, sin poder ser remolcada por una serie de fallos técnicos. En 2013 la Guardia Costera constató 16 violaciones graves en los dispositivos de contención del pozo para casos de emergencia. Shell anunció entonces que suspendía temporalmente las actividades de perforación mientras llevaba a cabo la reparación de las instalaciones. Sin embargo, en 2014 decidió cancelarlas definitivamente ante las pocas expectativas económicas de esta exploración y la decisión del Tribunal de Apelaciones para el Noveno Circuito mencionada, que dictaminó que el DoI había violado la legislación federal por no evaluar adecuadamente los efectos ambientales de estas explotaciones (Native Village of Point Hope v. Jewell, 2014). En diciembre de este año, Noble Drilling LLC, propietaria del buque de perforación, admitió ante la justica norteamericana la comisión de varios delitos relativos a la seguridad y la protección ambiental, por lo que fue sancionada con 12.2 millones de dólares (Federal District Court, Eastern District of Louisiana, In re Deepwater Horizon, 21 F.Supp.3d 657, 742, 2014). En 
nuevas medidas muy significativas. Por un lado, en julio de 2016, el DoI aprobó una nueva reglamentación, la Oil and Gas and Sulphur Operations on the Outer Continental Shelf - Requirements for Exploratory Drilling on the Arctic Outer Continental Shelf ${ }^{128}$, aplicable específicamente a las operaciones de perforación de la plataforma continental en los mares de Beaufort y Chukchi, en virtud del cual se exigen mayores garantías por parte de las compañías petroleras respecto a los controles y la planificación para la prevención, contención y respuesta ante el riesgo de vertidos de petróleo. Por el otro, en diciembre de 2016 se adoptó una nueva Executive Order presidencial ${ }^{129}$ que afecta la totalidad del Mar de Chukchi y de la mayor parte del Mar de Beaufort en Alaska y que en la práctica supone la retirada de estos territorios de futuros planes de arrendamiento, que solo autoriza en el área de Cook-Inlet.

No obstante, en la nueva línea del gobierno de Estados Unidos, el 28 de abril de 2017 el Presidente Trump emitió una nueva orden ejecutiva para revertir esta suspensión de los arrendamientos y eliminar todas las protecciones que la última orden del Presidente Obama proporcionaba a estas áreas ${ }^{130}$, una actuación que no tiene precedentes en el sistema jurídico estadounidense y que plantea ahora la conformidad jurídica de la acción presidencial. La OSCLA, en virtud de la cual se adoptaron ambas medidas, confiere la potestad al presidente para bloquear de forma indefinida las perforaciones petroleras y gasísticas en los espacios controlados por el gobierno federal, pero no contempla la posibilidad de revertir esta decisión mediante otra Executive Order. En principio, pues, la decisión del Presidente Obama tendría un carácter permanente y solo podría ser modificada por el Congreso, que podría bien decidir la nueva disponibilidad de los

enero de 2015, en una decisión muy atacada por los grupos ambientalistas, el DoI concedió a Shell una nueva autorización para la perforación en el Mar de Chukchi. En junio de 2015 la Corte de Apelaciones del Noveno Circuito rechazó la petición de un grupo de entidades para revocar la aprobación federal (Alaska Wilderness League y otros v. Jewell et al, No. 13-35866). Empezó su actividad en julio, pero dos meses después la compañía anunció la finalización de las perforaciones y su retirada del Mar de Chukchi, alegando que los resultados de las exploraciones no eran suficientemente satisfactorios.

12881 C.F.R. 46477, 15.7.2016.

129 The White House, Presidential Memorandum: Withdrawal of Certain Portions of the United States Arctic Outer Continental Shelf from Mineral Leasing, 20.12.2016, disponible online en https://obamawhitehouse.archives.gov/the-press-office/2016/12/20/presidential-memorandumwithdrawal-certain-portions-united-states-arctic.

130 The White House,Presidential Executive Order Implementing an America-First Offshore Energy Strategy, 28.4.2017, disponible enhttps:/www.whitehouse.gov/the-press-office/2017/04/28/presidentialexecutive-order-implementing-america-first-offshore-energy. 
arrendamientos en las aguas árticas, bien modificar la propia OSCLA para permitir que una nueva Executive Order de la Presidencia invalidara la anterior.

En cualquier caso, se abre ahora un periodo de incertidumbre y ésta será probablemente una cuestión que deberá resolver la Corte Suprema en un futuro próximo. De hecho, como respuesta a esta situación, en mayo de 2017 diversos grupos conservacionistas norteamericanos y comunidades indígenas de Alaska presentaron una demanda ante el Tribunal de Distrito de Alaska reclamando su anulación por entender que la decisión del Presidente Trump excede su autoridad constitucional y estatutaria y viola la legislación federal $^{131}$.

Incluso si puede revertirse la Executive Order de diciembre de 2016, es necesario que la administración Trump o el Congreso desarrollen una estrategia para reintegrar las áreas árticas excluidas en el programa de arrendamiento quinquenal. A este respecto, los hechos no dejan mucho lugar al optimismo. Paralelamente a la presentación de la demanda ante el Tribunal de Distrito de Alaska, el Partido Republicano ya ha introducido en el Senado una nueva propuesta legislativa, la Offshore Production and Energizing National Security Alaska Act (OPENS Alaska Act) para modificar la OSCLA y revocar la Executive Order de diciembre de $2016^{132}$. La política de hechos consumados de la nueva administración avanza irremediablemente y pocos días después de la adopción de la Executive Order por el Presidente Trump en abril de este año y conforme a su mandato, el gobierno federal inició también el proceso de revisión del actual programa de arrendamiento 2017-2022 y la reconsideración de los principios y las normas que afectan al desarrollo de la explotación de hidrocarburos en la plataforma continental $^{133}$. El primer paso para el desarrollo del nuevo plan quinquenal de la administración Trump ha sido la apertura de un periodo inicial de información pública y participación de cuarenta y cinco días, que finalizó el pasado 17 de agosto de $2017^{134}$.Parece, pues, imparable por ahora la apertura del Ártico de Alaska para el

\footnotetext{
${ }^{131}$ Alaska District Court, League of Conservation Voters, et al. v. Donald J. Trump, et al., $\mathrm{n}^{\circ}$ 3:17-cv00101, disponible online en http://earthjustice.org/sites/default/files/files/Complaint-arctic.pdf

${ }^{132}$ S.883-OPENS Alaska Act (2017-2018), disponible online en https://www.congress.gov/bill/115thcongress/senate-bill/883; Vid., "Murkowski and Sullivan Introduce Bill to Restart Alaska OCS Development", Republican News, 7.4. 2017.

${ }^{133}$ Order of the Secretay of Interior: America First Offshore Energy Strategy, $\mathrm{n}^{\circ}$ 3350, 1.5.2017, disponible en https://www.doi.gov/sites/doi.gov/files/press-release/secretarial-order-3350-offshore508.pdf.

${ }^{134}$ US Department of Interior, Bureau of Ocean Energy Management, Request for Information and Comments on the Preparation of the 2019-2024 National Outer Continental Shelf Oil and Gas Leasing
} 
desarrollo de la industria petrolera y gasística, que tendrá como efecto inmediato la posibilidad de nuevas perforaciones de petróleo y gas offshore en el $98 \%$ de sus aguas federales.

\section{CONSIDERACIONES FINALES}

El deshielo del Ártico como consecuencia del cambio climático presenta nuevas oportunidades para el desarrollo económico de la región. En particular, el acceso de la industria petrolera y gasística a las vastas reservas aún no exploradas constituye sin duda una de las perspectivas de futuro que despiertan un mayor interés comercial. Ello es así a pesar de la lejanía, la falta de infraestructuras, la presencia del hielo marino y los largos períodos oscuridad, un entorno único que dificulta la realización de esta actividad. De hecho, el desafío técnico que plantea la exploración y explotación de los hidrocarburos árticos, junto con un precio bajo del petróleo y la nueva agenda climática propiciada por el Convenio Marco de 1992, el Protocolo de Kioto de 1997 y el Acuerdo de Paris de 2015 han frenado fugazmente el interés por estos recursos. Aunque esta realidad ha templado el entusiasmo de algunas compañías petrolíferas, nada permite augurar por ahora el fin de la actividad extractiva en el Ártico. Al contrario, existe un cierto acuerdo en que en el futuro, -“cambio climático mediante”- habrá una mayor actividad de exploración y explotación, sino a corto, sí a medio o largo plazo.

No hay duda de que la exploración y explotación de los hidrocarburos en el Ártico va asociada a un riesgo extraordinario de dañar de forma irreversible un entorno único y extremadamente vulnerable. El ecosistema ártico es muy diverso y aloja a un número importante de comunidades autóctonas, lo que exige un desarrollo responsable y comprometido con el más alto nivel de protección ambiental. En este sentido, la degradación del Ártico constituye ya una seria amenaza para la sostenibilidad de las comunidades autóctonas y la protección del medio ambiente del conjunto del planeta.

A diferencia de la Antártida, la ausencia de un régimen integral de protección deja su gestión en manos de los Estados ribereños, siendo como son pocas aún las normas internacionales de obligatorio cumplimiento aplicables a esta región. El creciente interés comercial que despierta el Ártico, combinado con la particularidad de sus ecosistemas

Program MAA104000, Federal Register, Vol. 82 (126), 3.7.2017, disponible online en https://www.boem.gov/82-FR-30886/ 
naturales y la presencia de comunidades indígenas, exigen un modelo innovador y un marco legal adecuado, en el que la exploración y explotación de hidrocarburos, probablemente inevitable, debe ser muy limitada y responsable.

Estados Unidos, como potencia ártica, ha tenido una oportunidad única para combinar el ejercicio de sus poderes soberanos con la tarea de limitar los riesgos actuales para el frágil medio marino y terrestre del Ártico. La presidencia de Barack Obama ha intentado, con mayor o menor éxito, revertir una situación de desinterés general y colocar a la región ártica entre las prioridades nacionales. La Estrategia nacional para el Ártico de 2013, a la vez que confirmaba la posibilidad de nuevas oportunidades económicas significativas en la región, advertía también contra el desarrollo irrestricto de estos recursos. En esta línea, reclamaba una administración responsable basada en un enfoque de gestión integrado con el objetivo de promover una gestión sostenible y resiliente a largo plazo en la región.

En particular, el vertido de petróleo en el Golfo de México en 2010y las vicisitudes experimentadas por Shell en el Mar de Chukchi desde 2012 han tenido un impacto importante en el tratamiento de las actividades de exploración y explotación de hidrocarburos árticos, específicamente en lo que se refiere a la perforación en zonas ambientalmente más frágiles. De este modo, a lo largo de varios años se han producido actuaciones conducentes a reforzar algunos mecanismos para limitar el impacto de las actividades petrolíferas y gasísticas en la plataforma continental, como la reducción por el Congreso y el gobierno federal del alcance de los sucesivos programas de arrendamiento de los territorios federales, especialmente en ciertas áreas especialmente sensibles de Alaska. Así, a pesar del interés del gobierno federal por este potencial energético para la promoción del desarrollo económico de este territorio, en los últimos años tan solo se han concedido algunos arrendamientos federales en los mares de Beaufort y Chukchi, y de hecho, en la actualidad sólo el Mar de Beaufort tiene algún pozo de producción en funcionamiento ${ }^{135}$.

Sin embargo, es posible que la velocidad y la intensidad de este cambio no haya sido suficiente y sus resultados difícilmente vayan a permanecer en el tiempo. Por un lado, aunque ha coincidido momentáneamente con la reducción de las expectativas generadas y el hecho de que estos recursos no constituyen por ahora un elemento especialmente

${ }^{135}$ US Bureau of Ocean Energy Management, BP Exploration (Alaska) (BPXA)—Northstar, disponible online en http://www.boem.gov/About-BOEM/BOEM-Regions/Alaska-Region/Leasing-andPlans/Plans/BP-North-Star.aspx. 
significativo de la industria petrolífera estadounidense, esta acción no ha estado exenta de tensiones en torno al papel del gobierno federal y del gobierno estatal de Alaska con respecto a los arrendamientos para la exploración y explotación mar adentro. Por el otro, en estos años también se ha evidenciado un marco jurídico insuficiente, demasiado fragmentado y poco adaptado a las condiciones únicas de su medio marino ártico, en el que intervienen un complejo entramado de departamentos y agencias tanto a nivel federal como estatal que generalmente han ido actuando de manera reactiva ante situaciones de gran impacto ambiental. Aunque accidentes como los sufridos por el Exxon Valdez en 1989 en Alaska o por la plataforma del Deepwtare Horizon veinte años después en el Golfo de México no son frecuentes, la magnitud del riesgo requiere necesariamente una actitud de permanente cautela por parte de los gobiernos de los Estado ribereños del Océano Ártico, sin que quepa caer en la complacencia o el simple olvido de las consecuencias del último desastre a medida que su recuerdo se desvanece en la memoria.

\section{BIBLIOGRAFÍA}

ALCAIDE FERNÁNDEZ, J.,- CINELLI, C., "La cuestión ártica y el Derecho Internacional", Revista Española de Derecho Internacional, Vol. 61 (2), 2009, p. 381.

ARRUDA, G.M., "Arctic governance regime: The last frontier for hydrocarbons exploitation", International Journal of Law and Management, Vol. 57 (5), 2015, p. 498.

BIRD, K.J. et al., Circum-Arctic Resource Appraisal: Estimates of Undiscovered Oil and Gas North of the Arctic Circle, U.S. Geological Survey, 2008, disponible online en http://pubs.usgs.gov/fs/2008/3049.

BRATSPIES, R.M., “A Regulatory Wake-up Call: Lessons from BP's Deepwater Horizon Disaster", Golden Gate University Environmental Law Journal, Vol. 5, 2011, p. 7 .

BUDZIK, Ph., Arctic Oil and Natural Gas Potential, U.S. Department of Energy, Energy Information Administration, 2009, disponible online en https://www.eia.gov/analysis/studies/archive/2009/arctic/pdf/arctic_oil.pdf.

CAMPINS ERITJA, M., "The European Union and the North: Towards the Development of an EU Arctic Policy?, Ocean Yearbook, Vol. 27, 2013, p. 459. 
CAMPINS ERITJA, M., "Strengthening the European Union-Greenland's Relationship for Enhanced Governance of the Arctic", en LIU, N.; KIRK, E.; CHURCHILL, R.; AND HENRIKSEN, T., (Ed.), The European Union and the Arctic, Brill Academic Publishers, 2017, p. 65.

CANUEL, E., "Alaska and offshore hydrocarbon extraction. A legal and socioeconomic review", en PELAUDEIX, C.-BASSE, E.M., (Ed.), Governance of Arctic Offshore Oil and Gas, Routledge, 2017, p. 95.

CASPER, K.N., "Oil and Gas Development in the Arctic: Softening of Ice Demands Hardening of International Law", Natural Resources Journal, Vol. 49, 2009, p. 825.

CINELLI, C., "La cuestión ártica y la Unión Europea”, Revista Española de Relaciones Internacionales, Vol. 1, 2009, p. 138.

CINELLI, C., "La protección del medio ambiente ártico: Desarrollos recientes", en SOBRIDO PRIETO, M. (Ed.), Espacios polares y cambio climático: Desafíos jurídicointernacionales, Tirant lo Blanch, 2017, p. 185.

CONDE PÉREZ, E., "Delimitaciones marítimas y territoriales en el Ártico: Desarrollo y tendencias", Revista Española de Derecho Internacional, Vol. 68 (1), 2016, p. 235.

CONDE PÉREZ, E., "Geopolítica del Ártico: El derecho internacional ante los retos del cambio climático en la región", Cursos de Derecho Internacional y Relaciones Internacionales de Vitoria Gasteiz, 2014, p. 99.

CONDE PÉREZ, E., “Geopolítica del Ártico. Especial referencia a los intereses de España en la región ártica”, en Geopolítica del Ártico. Dos visiones complementarias. España-Singapur, Ministerio de Defensa, 2014, p. 15.

CONDE PÉREZ, E., YANEVA, Z.V., "The European Arctic Policy in progress”, Polar Science, Vol. 10, 2016, p. 441.

CONLEY, H., The New Foreign Policy Frontier: US interests and actors in the Arctic, Center for Strategic \& International Studies, 2013, disponible en https://narfu.ru/upload/medialibrary/8e7/2013_conley_newforeignpolfrontier_web_0_1_.pdf.

CONLEY, H., KRAUT, J., U.S. Strategic Interests in the Arctic. An Assessment of Current Challenges and New Opportunities for Cooperation, CSIS Europe Program, Center for Strategic \& International Studies, 2010, disponible online en http://csis.org/files/publication/100426_Conley_USStrategicInterests_Web.pdf. 
DLOUHY, J.A., "Big Oil Abandons \$2.5 Billion in U.S. Arctic Drilling Rights", Bloomberg, mayo 2016, disponible online https://www.bloomberg.com/news/articles/2016-05-10/big-oil-abandons-2-5-billion-inu-s-arctic-drilling-rights.

DUESUND, J.M., Arctic Offshore gas \& Oil Outlook, Rystad Energy, 2015, disponible online en https://www.rystadenergy.com/NewsEvents/PressReleases/arctic-offshoreoil,-gas-outlook.

EBINGER, CH., BANKS, J.P., SCHACKMANN, A., Offshore Oil and Gas Governance in the Arctic. A Leadership Role for the U.S., Brookings Institute, Policy Brief 14-01, 2014, disponible online en https://www.brookings.edu/wpcontent/uploads/2016/02/Offshore-Oil-and-Gas-Governance-web.pdf.

GULAS, S., DOWNTON, M., D'SOUZA, K., HAYDEN, K., WALKER, T.R., "Declining Arctic Ocean oil and gas developments: Opportunities to improve and environmental pollution control”, Marine Policy, Vol. 75, 2017, p. 53.

HENDERSON, J., LOE, J., The Prospects and Challenges for Arctic Oil Development, OIES Paper: WPM 54, Oxford Institute for Energy Studies, 2014.

HOAG, H., "Arctic Development Stalls with Tumbling Oil Prices", Arctic Deeply, enero 2016, disponible online en https://www.newsdeeply.com/arctic/articles/2016/01/22/arctic-development-stalls-withtumbling-oilprices.

HOSSAIN, K., MORRIS, K., "Protecting Arctic Ocean Marine Biodiversity in the Area Beyond National Jurisdiction. Plausible Legal Frameworks for Protecting High Arctic Waters", en ANDREONE, G., (Ed.), The Future of the Law of the Sea. Bridging Gaps Between National, Individual and Common Interests, Springer Open, 2017, p. 105

HOUCK, O.A., "Worst Case and the Deepwater Horizon Blowout: There Ought to Be a Law", Tulane Environmental Law Journal, Vol. 24 (1), 2010, p. 1.

HUEBERT, R. The United States Arctic Policy: The Reluctant Arctic Power, The School of Public Policy, University of Calgary, SPP Briefing Papers Focus on the United States. Vo. 2 (2), 2009, disponible online en http://policyschool.ucalgary.ca/files/publicpolicy/SPPBriefing-HUEBERTonline.pdf. HUEBERT, R., "Reinforcing Sovereignty, National Security and Circumpolar Cooperation", Northern Perspectives, Vol. 30 (1), 2006, p.7. 
HULTS, D., "Environmental Regulation at the Frontier: Government Oversight of Offshore Oil Drilling North of Alaska”, Environmental Law, Vol. 44, 2014, p. 761.

JEFFRIES, M.O., OVERLAND, J.E, PEROVICH,D.K., "The Arctic shifts to a new normal”, Physics Today, Vol. 66(10), 2013, p. 35.

JOKELA, J. (Ed.), Arctic Security Matters, EU Institute for Security Studies, 2015, disponible online en http: //www.iss.europa.eu/uploads/media/Report_24_Arctic_matters.pdf.

KALEN, S., "Cruise Control and Speed Bumps: Energy Policy and Limits for Outer Continental Shelf Leasing", Environmental \&Energy Law \& Policy Journal, Vol. 7, 2012, p. 156.

KEIL, K. "Spreading Oil, Spreading Conflict? Institutions Regulating Arctic Oil and Gas Activities” The International Spectator, Vol. 50 (1), 2015, p. 85.

KEIL, K., "The Arctic: A new region of conflict? The case of oil and gas", Cooperation and Conflict, Vol. 49 (2), 2014, p. 162.

KOIVUROVA, T., MOLENAAR, E.J., VANDERZWAAG, D.L., "Canada, the European Union, and Arctic Ocean Governance: A Tangled and Shifting Seascape and Future Directions”, en KOIVUROVA, T., CHIRCOP, A., FRANCKX, MOLENAAR, E.J., VANDERZWAAG, D.L. (Ed.), Understanding and Strengthening European Union-Canada Relations in Law of the Sea and Ocean Governance, Juridica Lapponica, Vol. 35, University of Lapland, 2009, p. 107.

KUERSTEN, A., "The Arctic Five Versus the Arctic Council”, Arctic Yearbook, 2016, p. 389.

LAJEUNESSE, A., "The New Economics of North American Arctic Oil", American Review of Canadian Studies, Vol. 43 (1), 2013, p. 107.

LINXWILER, J.D., "Federal Oil and Gas Leasing in Alaska", en Rocky Mountain Mineral Law Foundation (Ed.), Law on Federal Oil and Gas Leases, LexisNexis, 1985 $\begin{array}{lllll}\text { (puesta } & \text { al } & \text { dia } & \text { anual), } & \text { disponible }\end{array}$ https://www.google.es/search?q=Alaska+National+Interest+Lands+Conservation+Act+ AND+arctic+oil+sales\&ie $=$ utf-8\&oe $=$ utf-8\&client=firefox-b\&gfe_rd=cr\&ei=eqRWfMtjoPiBKakqKAM. 
MANERO SALVADOR, A., El deshielo del Ártico: Retos para el derecho internacional. La delimitación de espacios marinos y la protección y preservación del medio ambiente, Aranzadi-Thomson Reuters, 2011.

MILLERT, D.S., “Offshore Federalism: Evolving Federal-State Relations in Offshore Oil and Gas Development”, Ecology Law Quartlerly, Vol. 11, 1984, p. 401.

MOLENAAR, E.J., “Arctic Marine Shipping: Overview of the International Legal Framework. Gaps and Option”, Journal of Transnational Law \& Policy, Vol. 18, 2009, p. 289.

NORCHI, CH.H., “The Deepwater Horizon Incident”, Ocean and Coastal Law Journal, Vol. 16 (2), 2011, p. 245.

NORD, D.C., The Arctic Council: Governance Within the Far North, Routledge, 2016

O'ROURKE, R., Changes in the Arctic: Background and Issues for Congress, Congressional Research Service, 2016, disponible online en https://www.hsdl.org/?view\&did=796197.

O'ROURKE, R., Changes in the Arctic: Background and Issues for the Congress, Congressional Researchs Service, 2017, disponible online en http://fas.org/sgp/crs/misc/R41153.pdf.

OSHERENKO, G.-YOUNG, O., The Age of the Arctic: Hot Conflicts and Cold realities, Cambridge University Press, Cambridge, 2005.

OSOFSKY, H.M. "Multidimensional Governance and the BP Deepwater Horizon Oil Spill”, Florida Law Review, Vol. 63 (5), 2011, p. 1077.

OSOFSKY, H.M., SHADIAN, J., FECHTELKOTTER, S.L.,“Arctic Energy Cooperation",U.C. Davis Law Review, Vol. 49,2016, p. 1431.

PACHAURI, R.K., MEYER, L.A., Cambio climático 2014: Informe de síntesis. Contribución de los Grupos de trabajo I, II y III al Quinto Informe de Evaluación del Grupo Intergubernamental de Expertos sobre el Cambio Climático, IPCC, Ginebra, 2014.

PAREJA, P., "El Ártico como espacio de cooperación: Evolución, contenido y limites”, en SOBRINO HEREDIA, J.M., (Ed.), Mares y océanos en un mundo de cambio: Tendencias jurídicas, actores y factores, 2007, p. 883. 
RAZO, G.R, "The Seaweed Rebellion Revisited: Continuing Federal-State Conflict in OCS Oil and Gas Leasing”, Willamette Law Review, Vol. 20, 1984, p. 83.

REED, M.W., Shore and Sea Boundaries, Vol. 3, U.S. Department of Commerce, National Oceanic and Atmospheric Administration, Coastal Services Center, Office of Coast Survey - U.S. Government Printing Office, Washington, 2000.

RAYFUSE, R., "Melting Moments: The Future of Polar Oceans Governance in a Warming World", RECIEL, vol. 16(2), 2007, p. 196.

ROSSI, CH.R., "The club within the club: The challenge of a soft law framework in a global Arctic context", The Polar Journal, Vol. 5 (1), p. 8.

ROTHWELL, D.R., "The Arctic Environmental Protection Strategy and International Environmental Protection in the Far North", Yearbook of International Environmental Law, Vol. 6 (1), 1995, p. 65.

SANCHEZ RAMOS, B., "La institucionalización de la cooperación en el Ártico: Una mirada al Consejo Ártico", en SOBRIDO PRIETO, M. (Ed.), Espacios Polares y Cambio Climático: Desafíos jurídico-internacionales, Tirant lo Blanch, 2017, p. 139.

SCHOENBAUM, TH.J., "Liability for Damages in Oil Spill Accidents: Evaluating the USA and International Law Regimes in the Light of Deepwater Horizon", Journal of Environmental Law, Vol. 24 (3), 2012, p. 395.

SCHOENBAUM, TH.J., "The Deepwater Horizon Oil Spill in the Context of the Public International Law Regimes for the Protection of the Marine Environment: A Comparative Study", University of San Francisco Maritime Law Journal, Vol. 25, 2012-2013.

SOBRIDO PRIETO, M., “La gobernanza del Ártico”, en SOBRIDO PRIETO, M. (Ed.), Espacios polares y cambio climático: Desafíos jurídico-internacionales, Tirant lo Blanch, 2017, p. 105.

SPICER, W., "Deepwater Horizon Lessons for the Offshore", Dalhousie Law Journal, Vol. 37 (1), 2014, p. 205.

STĘPIEŃ, A., RASPOTNIK, A., “The EU's new Arctic Communication: Not-sointegrated, not so-disappointing?”, ArCticles, Arctic Centre Papers 1/2016, 2016.

VANDERZWAAG, D.L., "On the Road to Kingdom Come", en VANDERZWAAG, D.L. (Ed.), The Challenge of Arctic Shipping: Social, Environmental Assessment and Human Values, McGill-Queen University Press, 1990, p. 219. 
VINOGRADOV, S., "The Impact of the Deepwater Horizon: The Evolving International Legal Regime for Offshore Accidental Pollution Prevention, Preparedness, and Response", Ocean Development and International Law, Vol. 44 (4), 2013, p. 335.

WILlIS, M., The Arctic Council: Underpinning Stability in the Arctic, The Arctic Institute/Centre for Circumpolar Security Studies, 2013, disponible online en http://www.thearcticinstitute.org/the-arctic-council-underpinning/.

YOUNG, O., "Arctic in Play: Governance in a Time of Rapid Change", The International Journal of Marine and Coastal Law, Vol. 24, 2009, p. 423. 